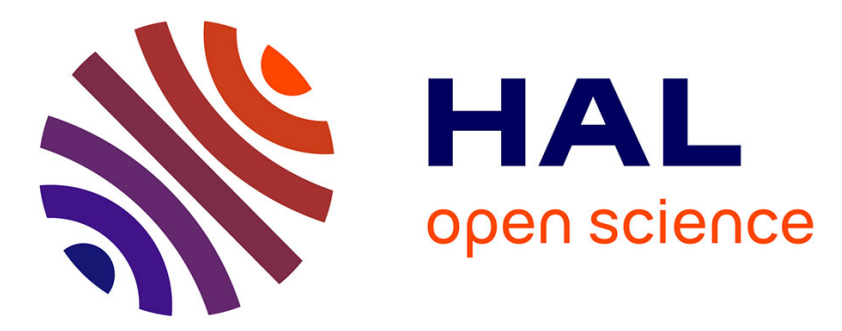

\title{
A nongray-wall emissivity model for the Wide-Band Correlated K-distribution method
}

\author{
Y. Liu, J. Zhu, G. Liu, J.L. Consalvi, Franklin Liu
}

\section{To cite this version:}

Y. Liu, J. Zhu, G. Liu, J.L. Consalvi, Franklin Liu. A nongray-wall emissivity model for the WideBand Correlated K-distribution method. International Journal of Heat and Mass Transfer, 2020, 159 (120095), 10.1016/j.ijheatmasstransfer.2020.120095 . hal-03189322

\section{HAL Id: hal-03189322 https://hal.science/hal-03189322}

Submitted on 28 Apr 2021

HAL is a multi-disciplinary open access archive for the deposit and dissemination of scientific research documents, whether they are published or not. The documents may come from teaching and research institutions in France or abroad, or from public or private research centers.
L'archive ouverte pluridisciplinaire HAL, est destinée au dépôt et à la diffusion de documents scientifiques de niveau recherche, publiés ou non, émanant des établissements d'enseignement et de recherche français ou étrangers, des laboratoires publics ou privés. 


\title{
A Nongray-Wall Emissivity Model for the Wide-Band Correlated K-
}

\section{Distribution Method}

\author{
Yuying LIU ${ }^{a}$, Jinyu ZHU ${ }^{a}$, Guanghai LIU ${ }^{a, *}$, Jean-louis CONSALVI ${ }^{b}$, Fengshan LIU \\ a. School of Energy and Power Engineering, Beihang University (BUAA), Beijing, 100191, China \\ b. Aix-Marseille Université, CNRS, IUSTI UMR 7343, 5 rue E. Fermi, 13013 Marseille, France \\ c. Measurement Science and Standards, National Research Council of Canada, 1200 Montreal Road, Ottawa, Ontario K1A 0R6, Canada \\ *Corresponding author: ghliu@buaa.edu.cn(G.LIU)
}

\begin{abstract}
The walls of combustion systems are usually assumed to be black or gray in radiative calculations, which may introduce large errors. The Planck-function-weighted emissivity is usually used as the gray-wall emissivity when using the Wide-Band Correlated K-distribution (WBCK) method. This approach can demonstrate good accuracy when the emissivity-based optimized band interval proposed by Solovjov (2013, JQSRT)(WBCK-1) is used. To improve efficiency without losing accuracy, a nongray-wall emissivity model is proposed. The accuracy of this model is first demonstrated for the emissivitybased band interval (WBCK-2). An absorption-coefficient-based optimized band interval is then proposed and the accuracy of this band interval coupled with the nongray-wall emissivity model (WBCK-3) is evaluated. The performance of these models is evaluated in three 1D isothermal and homogeneous cases bounded by fly-ash deposit, GH536, and soot deposit and a 3D fuel-air flame bounded by fly-ash deposit. The results show that WBCK-2 is more accurate than WBCK-1 when the number of bands is greater than 1. WBCK-3 can become more accurate than WBCK-1 and WBCK-2 when the number of bands is larger than 2, especially for low wall temperatures. Furthermore, it is sufficient to divide the spectrum into 3 bands for accurate predictions of radiative calculations.
\end{abstract}

Keywords: Radiative heat transfer; Wide-Band Correlated K-distribution; Emissivity; Nongray wall.

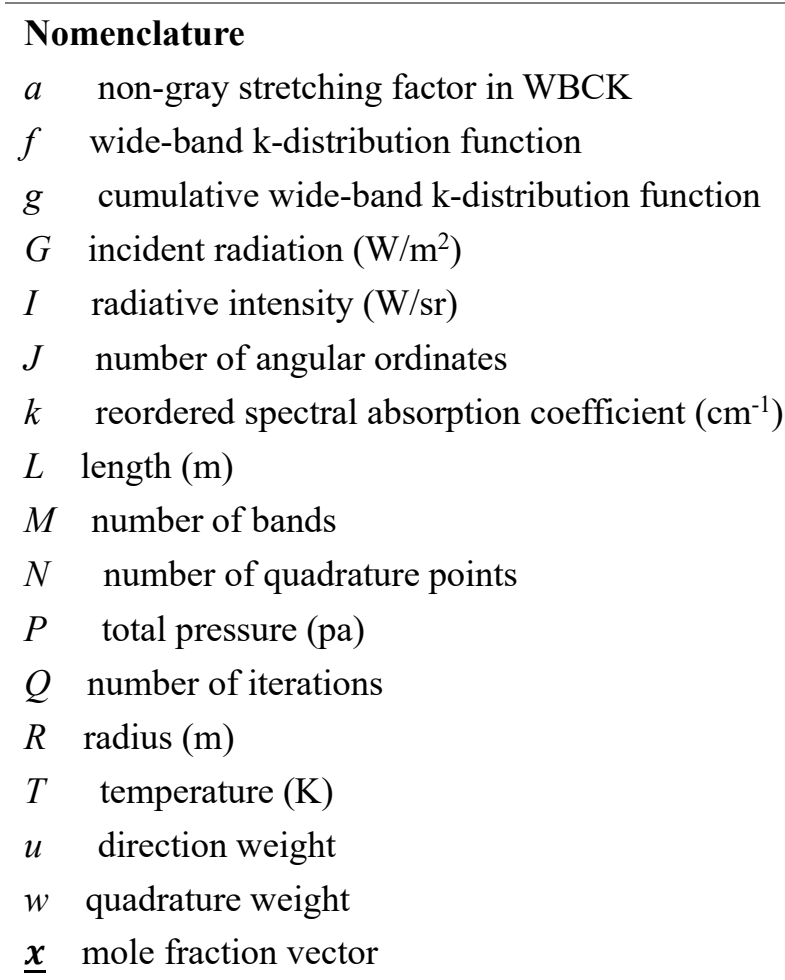

Greek symbols

\begin{tabular}{ll}
\multicolumn{2}{l}{ Subscripts } \\
$b$ & blackbody \\
$m$ & mth band \\
max & maximum \\
$l$ & lower \\
$n$ & $n$th quadrature point \\
$P$ & Planck \\
$\mathrm{j}$ & jth ordinates \\
$u$ & upper \\
$w$ & wall \\
0 & reference state
\end{tabular}

\begin{tabular}{|c|c|}
\hline \multicolumn{2}{|c|}{ Abbreviations } \\
\hline FSCK & Full-Spectrum Correlated K-distribution \\
\hline LBL & Line-By-Line \\
\hline \multicolumn{2}{|c|}{ MSMGFSK Multi-Scale Multi-Group FSK } \\
\hline NBCK & Narrow-Band Correlated K-distribution \\
\hline RTE & radiative heat equation \\
\hline SLW & Spectral-Line-based Weighted-sum-of-gray-gases \\
\hline
\end{tabular}




$\begin{array}{lll}\boldsymbol{\eta} & \text { wavenumber }\left(\mathbf{c m}^{-\mathbf{1}}\right) & \text { SNB } \quad \text { Statistical Narrow-Band } \\ \boldsymbol{\kappa} & \text { spectral absorption coefficient }\left(\mathbf{c m}^{-\mathbf{1}}\right) & \text { WBCK Wide Band Correlated-K } \\ \boldsymbol{\phi} & \text { vector of thermodynamic state variables } & \text { WSGG Weighted-Sum-of-Gray-Gases } \\ \boldsymbol{\delta} & \text { Dirac-delta function } & \\ \boldsymbol{\varepsilon} & \text { emissivity } & \end{array}$

\section{Introduction}

Radiative heat transfer plays an important role in high-temperature industrial combustion systems, such as coal-fired boilers and gas turbine combustors [1]. Most studies assume that the walls of combustors are black or gray in radiative transfer calculations for simplicity in spite of the fact that the wall materials have different spectral emissivity. This simplification may introduce large errors in the prediction of radiative source term and heat flux [2], which may strongly affect the predicted performance of the combustion devices in terms of thermal protection [3] and pollutant emissions [4]. Therefore, comprehensive and accurate radiative models are necessary for accurately predicting radiative heat transfer in practical problems involving nongray walls and for optimal design of combustion devices.

Radiative heat transfer models with different spectral resolutions, such as the Line-By-Line method (LBL), narrow-band models [5]-[], wide-band models [9], [] and full-spectrum models [8], 11]-13], provide different accuracy and computational efficiency [14]. The benchmark LBL method is rarely applied to practical applications due to the huge requirement in computing resources [15]. Narrow-band models, such as the Statistical Narrow-Band method (SNB) [5], ], the Statistical Narrow-Band Correlated-K method (SNBCK) [7] and the Narrow-Band Correlated K-distribution method (NBCK) [8], demonstrate the best accuracy in comparison to LBL because of small changes in spectral emissivity within a narrow spectral range for both gases and a solid wall. Although the number of radiative transfer equations (RTEs) of narrow-band models is much less than that of LBL, the computing resources required for solving narrow-band RTEs are still unacceptable in practical applications. Therefore, various computationally more efficient wide-band models and full-spectrum models have been developed. Full-spectrum models, such as the Full-Spectrum Correlated K-distribution method (FSCK) [8], the Spectral-Linebased Weighted-Sum-of-Gray-Gases method (SLW) [11] and the Weighted-Sum-of-Gray-Gases method (WSGG) [13], provide the highest computational efficiency at the cost of some accuracy for practical problems involving nongray walls [16], 17]. Wide-band models have a great potential for achieving the best compromise between efficiency and accuracy for practical problems with nongray walls. For example, Solovjov et al. [16] proposed a method to optimize band interval according to the spectral emissivity for the expansion of SLW named the cumulative wavenumber model, in which only a few RTEs need to be solved. Fonseca et al. [17] separated gray gases into several groups according to a presumed emissivity, followed by solving RTE with the WSGG method. The banded method was employed by Bordbar et al. [18], 19] to solve such problems as well. It was shown that those methods provide a good compromise between accuracy and efficiency for nongray wall problems.

The Planck-function-weighted emissivity at the wall temperature is usually used as the gray-wall emissivity to ensure the conservation of radiative energy at the wall [14]. This gray-wall emissivity model can achieve good accuracy when combined with the emissivity-based optimized band interval mentioned above [16] for nongray wall problems too. To further improve computational efficiency without significantly losing accuracy, some methods have been proposed recently. For instance, Fonseca et al. [20] used a radiating medium mean temperature, rather than the wall temperature, as the Planck temperature to obtain the gray-wall emissivity. Liu et al. [21] proposed a grouping strategy to improve the correlation between the absorption spectrum of the radiating medium and the emission spectrum of the nongray wall and then solved RTE with the Multi-Scale Multi-Group FSK method (MSMGFSK). It was shown that these two methods can demonstrate good accuracy and computational efficiency.

The objective of this work is to propose a nongray-wall emissivity model for the Wide-Band Correlated K-distribution (WBCK) method and compare this nongray-wall emissivity model with, on one hand, benchmarks solutions obtained from LBL calculations and, on the other hand, the gray-wall one, i.e., the Planck-function-weighted emissivity, in three 1D isothermal and homogeneous cases and a 3D fuel-air flame. All the 1D test cases are bounded by nongray walls composed of three typical materials found in industrial combustion devices, namely fly-ash deposit, GH536, and soot deposit, while the 
3D case is bounded by walls coated with the soot deposit. Similar to the treatments of Solovjov et al. [16] and Fonseca et al. [17], the present WBCK method is formulated by generating the Planck-function-weighted wide-band k-distribution. This article is organized as follows. The second section presents the theoretical background. The third and fourth sections are devoted to the comparison of different models in three 1D cases and one 3D fuel-air flame, respectively. Section 5 summarizes the conclusions drawn from the present work.

\section{Theoretical background}

\subsection{WBCK RTE}

The spectral RTE in participating and non-scattering media can be written as:

$\frac{d I_{\eta}}{d s}=\kappa_{\eta}(\underline{\phi})\left[I_{b \eta}(T)-I_{\eta}\right]$

where $\eta$ is the wavenumber, $I_{\eta}$ is the spectral radiative intensity along path $s, I_{b \eta}$ is the spectral Planck function, $\kappa_{\eta}$ is the spectral absorption coefficient, and $\underline{\phi}$ is the vector of local state variables consisting of temperature, $T$, total pressure, $P$, and mole fraction vector, $\underline{x}$.

Similar to the full-spectrum k-distribution function, the wide-band k-distribution function, $f_{m}$, which is weighted by the Planck function, is defined as [8][8]:

$f_{\mathrm{T}_{P}, \underline{\phi}, m}\left(k_{m}\right)=\frac{1}{I_{b, m}\left(\mathrm{~T}_{P}\right)} \int_{\eta_{l, m}}^{\eta_{u, m}} I_{b \eta}\left(\mathrm{T}_{P}\right) \delta\left(k_{m}-\kappa_{\eta}(\underline{\phi})\right) \mathrm{d} \eta$

where $\eta_{u, m}$ and $\eta_{l, m}$ are the upper and lower limits of $m$ th band, $I_{b, m}\left(\mathrm{~T}_{P}\right)\left(=\int_{\eta_{l, m}}^{\eta_{u, m}} I_{b \eta}\left(\mathrm{T}_{P}\right) \mathrm{d} \eta\right)$ is the spectrally-integrated Planck function of the $m$ th band.

The cumulative wide-band k-distribution function of the $m$ th band, $g_{m}$, which represents the fraction of spectrum whose spectral absorption coefficient is lower than an absorption coefficient variable $k_{m}$, is expressed as:

$g_{T_{P}, \underline{\phi}, m}\left(k_{m}\right)=\int_{0}^{k_{m}} f_{T_{P}, \underline{\phi}, m}\left(k_{m}^{\prime}\right) d k_{m}^{\prime}$

Thus, the cumulative function $g_{m}$ is a monotonically increasing function varying between 0 and 1 .

The RTE in the wavenumber-space can be converted into the reordered form in the reference $g_{0}$-space by multiplying with $\frac{\delta\left(k_{m}-\kappa_{\eta}\left(\underline{\phi}_{0}\right)\right)}{f_{T_{0}, \underline{\phi}_{0}, m}\left(k_{m}\right)}$, followed by integrating over all the wavenumbers belonging to the $m$ th band:

$\frac{d I_{g_{0}, m}}{d s}=k_{m}^{*}\left(g_{0}\right)\left[a_{m}\left(g_{0}\right) I_{b, m}(T)-I_{g_{0}, m}\right]$

where the subscript 0 refers to the reference state. In Eq. (4), $I_{g_{0}}$ and the non-gray stretching factor, $a_{m}\left(g_{0}\right)$, are expressed as:

$I_{g_{0}, m}=\frac{\int_{\eta_{l, m}}^{\eta_{u, m}} I_{\eta} \delta\left(k_{m}-\kappa_{\eta}\left(\underline{\phi}_{0}\right)\right) d \eta}{f_{T_{0}, \phi_{0}, m}\left(k_{m}\right)}$

$a_{m}\left(g_{0}\right)=\frac{f_{T, \underline{\phi}_{0}, m}\left(k_{m}\right)}{f_{T_{0}, \underline{\underline{p}}_{0}, m}\left(k_{m}\right)}=\frac{d g_{T, \underline{\phi}_{0}, m}\left(k_{m}\right)}{d g_{T_{0}, \underline{\phi}_{0}, m}\left(k_{m}\right)}$

The conventional correlated reordered absorption coefficient scheme proposed by Modest et al. [8] is used here to obtain the correlated reordered absorption coefficient $k_{m}^{*}$ using the following explicit equation:

$g_{T_{0}, \underline{\phi}, m}\left(k_{m}^{*}\right)=g_{0}$

Because the reordered absorption coefficient, $k_{m}^{*}$, is a monotonically increasing function of $g_{0}$, the total radiative intensity, $I$, can be obtained using a $N$-point quadrature scheme:

$I=\sum_{m=1}^{M} \sum_{n=1}^{N} w_{n} I_{g_{0}, m, n}$

where $M$ and $N$ are the number of bands and quadrature points, respectively, and $w_{n}$ is the weight of the $n$th quadrature point. It should be pointed out that the WBCK method degenerates to FSCK when $M=1$. 


\subsection{WBCK boundary conditions}

In this study, it is assumed that the walls reflect radiation diffusely and such an assumption has been used in many numerical studies[22], []. Consequently, the boundary condition of RTE in the wavenumber-space (Eq. (1)) is given as

$I_{\eta, w}=\underbrace{\varepsilon_{\eta} I_{b \eta}\left(T_{w}\right)}_{\text {emission term }}+\underbrace{\left(1-\varepsilon_{\eta}\right) \frac{1}{\int_{\vec{n}} \cdot \vec{s}<0} I_{\eta}|\vec{n} \cdot \vec{s}| d \Omega}_{\text {reflection term }}$

where $\varepsilon_{\eta}$ is the spectral emissivity of the wall, $\vec{n}$ is the unit vector normal to the wall and towards the medium, and the subscript $w$ refers to the wall.

\subsubsection{Gray-wall emissivity model}

In most previous studies, the walls are assumed to be black or gray in radiative calculations [22], ], i.e., the wavenumber dependence of wall emissivity is completely neglected. Similar to the derivation of Eq. (4), the boundary condition in the reference $g_{0}$-space can be given by:

$I_{g_{0}, m, w}=\underbrace{\varepsilon_{m} a_{m, w}\left(g_{0}\right) I_{b, m}\left(T_{w}\right)}_{\text {emission term }}+\underbrace{\left(1-\varepsilon_{m}\right) \frac{1}{\pi} \int_{\vec{n} \cdot \vec{s}<0} I_{g_{0}, m}|\vec{n} \cdot \vec{s}| d \Omega}_{\text {reflection term }}$

The Planck-function-weighted emissivity at the wall temperature is used as the gray-wall emissivity, which is expressed as [14]

$\varepsilon_{m}=\frac{1}{I_{b, m}\left(\mathrm{~T}_{w}\right)} \int_{\eta_{l, m}}^{\eta_{u, m}} I_{b \eta}\left(\mathrm{T}_{w}\right) \varepsilon_{\eta} \mathrm{d} \eta$

\subsubsection{Nongray-wall emissivity model}

In practical applications, the emissivity of walls normally varies with wavenumber. In this case, the emission term in Eq. (9) can be integrated over a wide band as

$\int_{\eta_{l, m}}^{\eta_{u, m}} \frac{\varepsilon_{\eta} I_{b \eta}\left(T_{w}\right) \delta\left(k_{m}-\kappa_{\eta}\left(\underline{\phi}_{0}\right)\right)}{f_{T_{0}, \underline{\phi}_{0}, m}\left(k_{m}\right)} d \eta=\int_{\eta_{l, m}}^{\eta_{u, m}}\left[\frac{\varepsilon_{\eta} I_{b \eta}\left(T_{w}\right) \delta\left(k_{m}-\kappa_{\eta}\left(\underline{\phi}_{0}\right)\right)}{f_{T_{w}, \underline{\phi}_{0}, m}\left(k_{m}\right)} \cdot \frac{f_{T_{w}, \underline{\underline{p}}_{0}, m}\left(k_{m}\right)}{f_{T_{0}, \underline{\phi}_{0}, m}\left(k_{m}\right)}\right] d \eta=\varepsilon_{m}^{*}\left(g_{0}\right) a_{m, w}\left(g_{0}\right) I_{b, m}\left(T_{w}\right)$

where $\varepsilon_{m}^{*}\left(g_{0}\right)$ is expressed as:

$\varepsilon_{m}^{*}\left(g_{0}\right)=\frac{1}{f_{T_{w}, \underline{\phi}_{0}, m}\left(k_{m}\right)} \cdot \frac{1}{I_{b, m}\left(T_{w}\right)} \cdot \int_{\eta_{l, m}}^{\eta_{u, m}} \varepsilon_{\eta} I_{b \eta}\left(T_{w}\right) \delta\left(k_{m}-\kappa_{\eta}\left(\underline{\phi}_{0}\right)\right) d \eta$

Therefore, the boundary condition in the reference $g_{0}$-space can be rewritten as

$I_{g_{0}, m, w}=\varepsilon_{m}^{*}\left(g_{0}\right) a_{m, w}\left(g_{0}\right) I_{b, m}\left(T_{w}\right)+\left(1-\varepsilon_{m}^{*}\left(g_{0}\right)\right) \frac{1}{\pi} \int_{\vec{n} \cdot \vec{s}<0} I_{g_{0}, m}|\vec{n} \cdot \vec{s}| d \Omega$

It is easy to anticipate that it is important to account for the nongray-wall emissivity when the emission term of Eq. (9) is much larger than the reflection term. However, for most combustion devices, the reflection term can also be important. Therefore, the importance of considering the nongray-wall emissivity needs to be evaluated under different wall and medium thermal conditions.

\subsection{Methods for determining the band interval of WBCK}

\subsubsection{Emissivity-based method}

The optimized band interval method proposed by Solovjov et al. [16] is obtained through minimization of the following objective function

$\sum_{m=1}^{M} \int_{\eta_{l, m}}^{\eta_{u, m}}\left|\varepsilon_{\eta}-\varepsilon_{m}\right| I_{b \eta}\left(\mathrm{T}_{w}\right) d \eta \rightarrow \min$

where the band emissivity, $\varepsilon_{m}$, is calculated by Eq. (11). In this study, $100 \mathrm{~cm}^{-1}$ is chosen as the band variation step to obtain the optimized band interval for simplicity. 


\subsubsection{Absorption-coefficient-based method}

The selection of emissivity-based method (Eq. (15)) may become inaccurate for low temperature walls. Taking the virtual spectral absorption coefficients and spectral emissivity distribution along the wavenumber shown in Fig. 1 as an example, supposing that there are two identical peak spectral absorption coefficients over the entire spectrum locating at $2500 \mathrm{~cm}^{-1}$ and $7500 \mathrm{~cm}^{-1}$, and the spectral emissivity increases linearly from 0 to 1 for the wavenumber of $0-10000 \mathrm{~cm}^{-1}$. It can be seen that the spectral emissivity at the peaks of spectral absorption coefficient are 0.25 and 0.75 , as shown in the left frame of Fig. 1(a). The emissivity-based method (Eq. (15)) for a wall temperature of $500 \mathrm{~K}$ decomposes the spectrum into two bands with the spectral ranges of $0-1400 \mathrm{~cm}^{-1}$ and $1400-10000 \mathrm{~cm}^{-1}$. The right frame of Fig. 1(a) shows that the correlated reordered absorption coefficient in the $g_{0}$ space, $k_{m}^{*}$, of the $2 n d$ band is 0 when $\mathrm{g}_{0}<1$, and is equal to the value of peak absorption coefficient in the left frame of Fig. 1(a) for $\mathrm{g}_{0}=1$. The nongray-wall emissivity in the $g_{0}$ space, $\varepsilon_{m}^{*}$, of the $2 n d$ band is 0 when $\mathrm{g}_{0}<1$, and is equal to about 0.25 for $\mathrm{g}_{0}=1$ according to Eq. (13) due to the Planck function weight. This shows clearly that the emissivity-based method introduces large errors for prediction of emissivity at the large absorption coefficients.

To improve the accuracy of the nongray-wall emissivity for large absorption coefficients that correspond to large reference $g_{0}$, an absorption-coefficient-based method is proposed here. The main idea of the proposed method is to put large absorption coefficients at different wavenumbers into different wide bands, as show in Fig. 1(b). If the two peak absorption coefficients are divided into two different bands, typically $0-5000 \mathrm{~cm}^{-1}$ and $5000-10000 \mathrm{~cm}^{-1}$, then $\varepsilon_{m}^{*}$ of the $1 s t$ and $2 s t$ band is equal to 0.25 and 0.75 when $\mathrm{g}_{0}=1$ respectively, which is much more accurate than that of emissivity-based method. For practical radiating medium, the spectral absorption coefficients of $\mathrm{CO}_{2}$ are mainly concentrated in 4 spectral positions, the proposed band interval determination method may provide good accuracy.

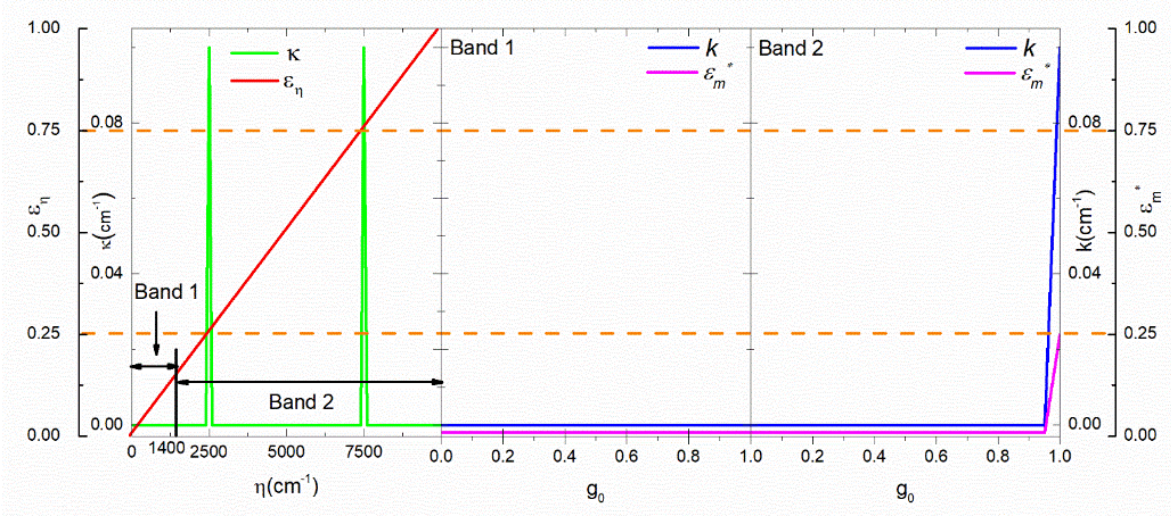

(a)

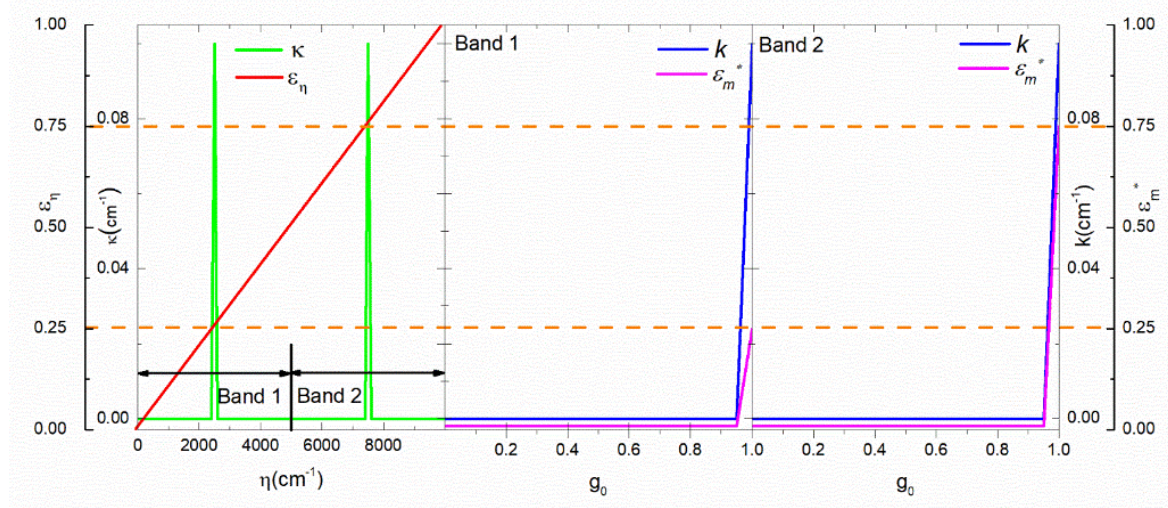

(b)

Fig. 1 Schematic diagram of band interval determination method.

(a) Emissivity-based band interval (b)Absorption-coefficient-based band interval

The optimization procedure is illustrated in Fig. 2, which is for $10 \% \mathrm{CO}_{2}$ (the left frame) and $10 \% \mathrm{H}_{2} \mathrm{O}$ (the right frame) at $2500 \mathrm{~K}$ and $1 \mathrm{~atm}$ as examples. In these two examples, it is assumed that the wall surface emissivity increases linearly between 
$0 \sim 1$ at a wavenumber of $0 \sim 10000 \mathrm{~cm}^{-1}$. The entire spectrum is divided into 4 bands.

Firstly, the narrow-band mean absorption coefficients over spectral bands of $200 \mathrm{~cm}^{-1}$ is calculated by Eq. (16) and the four maximum absorption coefficients, $A_{m}$, is marked in order in its magnitude.

$\kappa_{m}^{\prime}=\frac{1}{\eta_{u, m}-\eta_{l, m}} \int_{\eta_{l, m}}^{\eta_{u, m}} \kappa_{\eta} \mathrm{d} \eta$

Secondly, the wavenumber delimiters of all wide bands, $P_{m}$, are determined as the spectral locations of the minimum absorption coefficient between the adjacent maximum absorption coefficients, $B_{m}$, as show in Fig. 2(a) and Fig. 2(b), which takes the third band with an absorption coefficient greater than $0.1 \mathrm{~cm}^{-1}$ (marked by blue dashed line and related vertical pink lines) as an example. It can be seen that the wall emissivity in the wavenumber-space lies in a very small range of $0.34 \sim 0.37$ for Fig. 2(a) or $0.33 \sim 0.37$ for Fig. 2(b). The relatively high concentration of large absorption coefficients in each wide band makes the associated spectral wall emissivity vary weakly, which results in the reordered wall emissivity is very close to the true value in wavenumber space at larger reference $\mathrm{g}_{0}$ positions.

The reason for choosing the spectral bands of $200 \mathrm{~cm}^{-1}$ as narrow band rather than a narrower one, such as $100 \mathrm{~cm}^{-1}$, is that the profile of the narrow-band mean absorption coefficients over $200 \mathrm{~cm}^{-1}$ is smoother than over $100 \mathrm{~cm}^{-1}$, which helps locate the $A_{m}$ and $B_{m}$. However, it should be pointed out that the choice of narrow-band width in Eq. (16) is not critical since it only slightly affects the wavenumber between two adjacent wide bands.

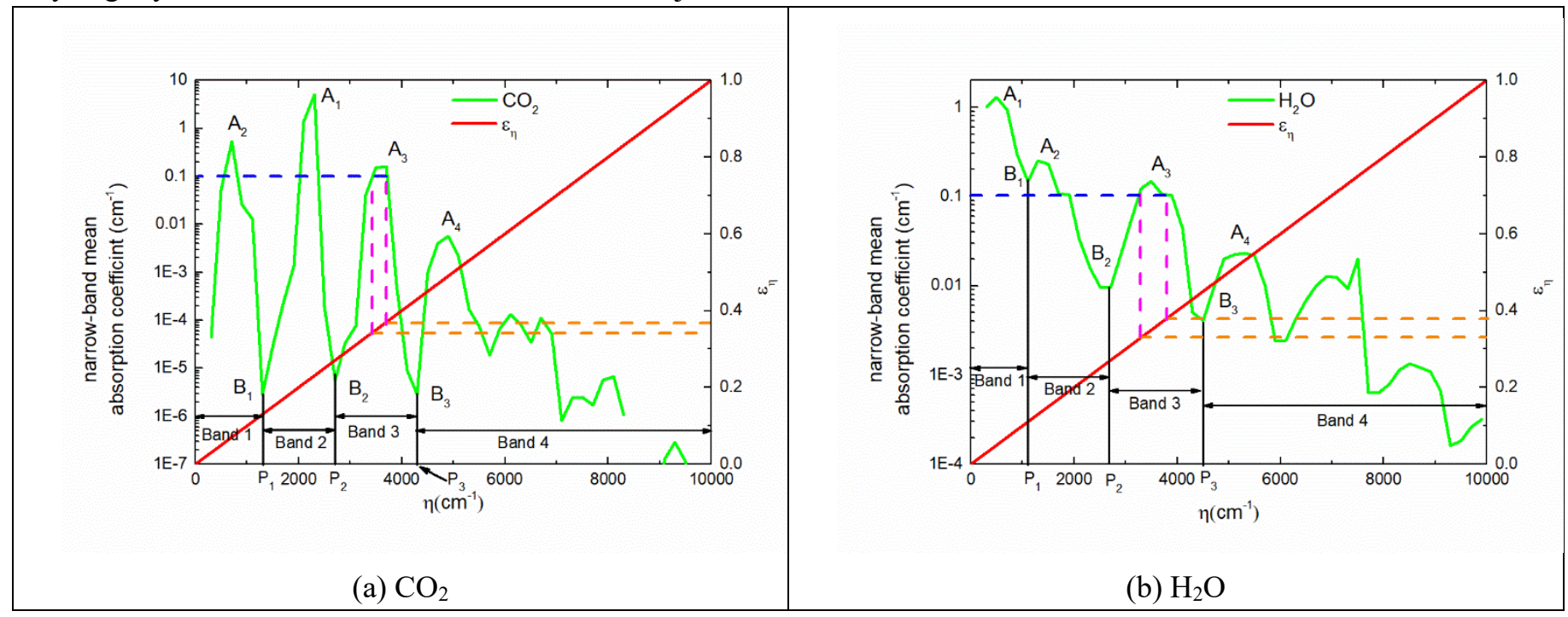

Fig. 2 Schematic diagram to illustrate the absorption-coefficient-based band interval.

Hereafter, the WBCK model with the emissivity-based band interval and the gray-wall emissivity model is named as WBCK-1. The WBCK model with the emissivity-based band interval and the nongray-wall emissivity model is named as WBCK-2 and the WBCK model with the absorption-coefficient-based band interval and the nongray-wall emissivity model is named as WBCK-3. It is noticed that WBCK-2 and WBCK-3 are identical when only 1-band $(M=1)$ is used.

\section{Results and discussion of one-dimensional cases}

It is natural to expect that different boundary conditions have different impacts on the predicted radiative source term and heat flux. Here three typical combustor surface materials with different spectral emissivities are considered to compare the accuracy of WBCK-1, WBCK-2 and WBCK-3, including GH536 at $1000 \mathrm{~K}$ [25], fly-ash deposit at $1093 \mathrm{~K}$ [26], and soot deposit with a thickness of $1 \mu \mathrm{m}$ [27] (hereafter named as BC-1, BC-2 and BC-3, respectively). Fly-ash deposit, which is typically found in coal-fired boilers, is a non-metallic material. Its spectral emissivity decreases with increasing the wavenumber, as shown in Fig. 3 . GH536 [25], as one of high-temperature alloys, is usually applied to gas turbine combustion chambers. Its spectral emissivity increases smoothly with increasing the wavenumber, Fig. 3. In hydrocarbon fuel combustion systems, soot deposit is often seen on the walls. Its spectral emissivity increases as the wavenumber increases as well, Fig. 3. The accuracy of WBCK-1, WBCK-2 and WBCK-3 with one (i.e., FSCK), two, and four bands is investigated, and the 
influence of the number of bands will also be discussed later.

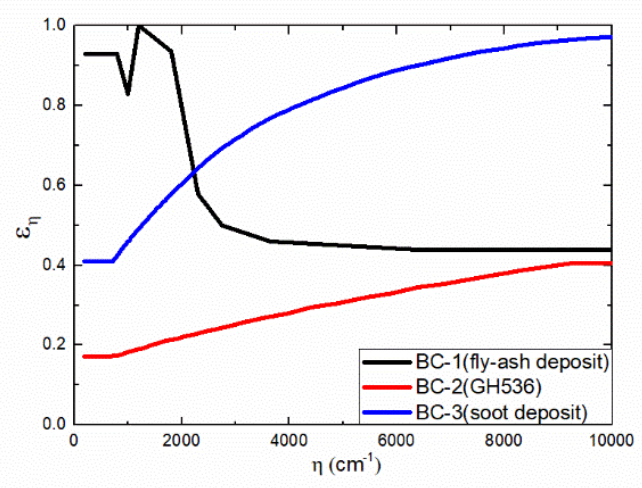

Fig. 3 The spectral emissivity of fly-ash deposit at 1093 K [26], GH536 at 1000 K [25], and soot deposit with a thickness of $1 \mu \mathrm{m}$ [27].

In this section, three one-dimensional isothermal and homogeneous cases are investigated, which includes a pure $\mathrm{CO}_{2}$ case, a pure $\mathrm{H}_{2} \mathrm{O}$ case, and a $\mathrm{CO}_{2}-\mathrm{H}_{2} \mathrm{O}$ mixture case, as shown in Table 1. The discrete-ordinates method (DOM) along with the $\mathrm{S} 8$ angular discretization scheme [14] is used to solve RTE. The spectral absorption coefficients of radiating gases are derived from the HITEMP 2010 database [29] and the HAPI code [30]. The range of wavenumber covers from $200 \mathrm{~cm}^{-1}$ to 10000 $\mathrm{cm}^{-1}$ and the wavenumber interval is set to $0.02 \mathrm{~cm}^{-1}$ in LBL calculations, which has been proven to be a good compromise between accuracy and efficiency [15]. The 32-point Gauss-Chebyshev scheme is selected to reduce the error induced by quadrature scheme. For both the gray and non-gray boundary conditions, iteration is necessary in solving the RTEs due to reflection of incident radiative heat flux at walls. The convergence condition for all the bands is

$\max \left(\frac{G_{m}^{Q}-G_{m}^{Q-1}}{G_{m}^{Q}}\right)<10^{-6}$

where $Q$ is the number of iterations and $G_{m}$ is incident radiation of the $m$ th band evaluated as

$G_{m}^{Q}=\sum_{n=1}^{N} \sum_{j=1}^{J} w_{n} u_{j} I_{g_{0}, m, n, j}$

where $u_{j}$ is weight of the $j$ th ordinate and $J$ is the number of angular ordinates.

Table 1. Description of the thermal conditions in the three one-dimensional cases.

\begin{tabular}{|c|c|c|c|c|c|c|c|}
\hline Case & $\begin{array}{c}\text { Number of } \\
\text { subcases }\end{array}$ & $\begin{array}{l}\text { Length L } \\
\text { (m) }\end{array}$ & $\begin{array}{c}\text { Internal } \\
\text { temperature }(\mathrm{K})\end{array}$ & $\begin{array}{l}\text { Wall temperature } \\
\qquad(\mathrm{K})\end{array}$ & $x_{\mathrm{CO}_{2}}$ & $x_{\mathrm{H}_{2} \mathrm{O}}$ & $\begin{array}{l}\text { Boundary } \\
\text { condition }\end{array}$ \\
\hline \multirow{3}{*}{1} & (a) & & & & & & BC-1 \\
\hline & (b) & 1 & 2500 & $300-2000$ & 0.1 & 0 & $\mathrm{BC}-2$ \\
\hline & (c) & & & & & & $\mathrm{BC}-3$ \\
\hline \multirow{3}{*}{2} & (a) & & & & & & BC-1 \\
\hline & (b) & 1 & 2500 & $300-2000$ & 0 & 0.1 & BC-2 \\
\hline & (c) & & & & & & BC-3 \\
\hline \multirow{3}{*}{3} & (a) & & & & & & BC-1 \\
\hline & (b) & 1 & 2500 & $300-2000$ & 0.1 & 0.2 & BC-2 \\
\hline & (c) & & & & & & $\mathrm{BC}-3$ \\
\hline
\end{tabular}

Three metrics are used to evaluate the accuracy of the three WBCK models, including the local relative error of radiative source term $(\operatorname{LE}(\mathrm{x}))$, the total absolute relative error of radiative source term (TE), and the error of heat flux at the right wall 
(EF). These quantities are defined as:

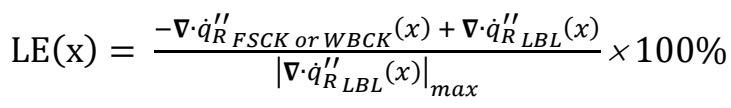

$\mathrm{TE}=\int_{0}^{L} \mid-\boldsymbol{\nabla} \cdot \dot{q}_{R}^{\prime \prime}{ }_{F S C K}$ or $W_{B C K}(x)+\boldsymbol{\nabla} \cdot \dot{q}_{R_{L B L}}^{\prime \prime}(x)\left|d x / \int_{0}^{L}\right| \boldsymbol{\nabla} \cdot \dot{q}_{R_{L B L}}^{\prime \prime}(x) \mid d x$

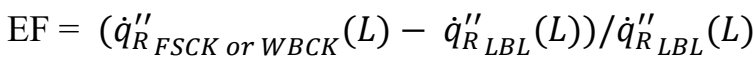

where $L$ is the length of computational domain.

\subsection{The pure $\mathrm{CO}_{2}$ case}

Firstly, a pure $\mathrm{CO}_{2}$ case (Case 1) is considered, as shown in Table 1 . The gaseous medium bounded by the two walls consists of $10 \% \mathrm{CO}_{2}$ (balanced by $\mathrm{N}_{2}$ ) at $2500 \mathrm{~K}$ and $1 \mathrm{~atm}$, while the wall temperature is varied from $300 \mathrm{~K}$ to $2000 \mathrm{~K}$. Figure 4 shows the variation of TE with the wall temperature. From the top frame of Figure 4, it can be seen that TE of 1-band WBCK1 decreases firstly as the wall temperature increases. After reaching the minimum at about $1400 \mathrm{~K}, 1100 \mathrm{~K}$ and $1000 \mathrm{~K}$ for $\mathrm{BC}-1, \mathrm{BC}-2$, and BC-3, respectively, it increases as the wall temperature further increases. In contrast, TE of 1-band WBCK2 (or WBCK-3) decreases firstly with increasing the wall temperature and then remains very small (using BC-1) or negligible (using $\mathrm{BC}-2$ and $\mathrm{BC}-3$ ) at sufficiently high wall temperatures.

The values of TE of 2- and 4-band WBCK-1 display different trends for different wall materials. For BC-1 and BC-3, TE of 2- and 4-band WBCK-1 drops sharply with increasing the wall temperature till around $500 \mathrm{~K}$, and then oscillates around a certain low value at higher wall temperatures, as shown in the middle and bottom rows of Figures 4(a) and 4(c). For BC-2, TE of 2- and 4-band WBCK-1 first decreases quickly to a minimum around 800 to $1000 \mathrm{~K}$ wall temperature but then increases quickly at a similar rate with increasing the wall temperature. In contrast, the TE values of 2- and 4-band WBCK-2 first decrease quickly with increasing wall temperature till around $500 \mathrm{~K}$ and then remain nearly constant close to zero with further increasing the wall temperature for all the three surface materials. In terms of TE, the accuracy of 2-band WBCK-3 is almost the same as 2-band WBCK-2, while the accuracy of 4-band WBCK-3 remains excellent regardless of the wall temperatures. In conclusion, 1-, 2- and 4-band WBCK-2 and WBCK-3 are much more accurate than their WBCK-1 counterparts, 2-band WBCK-2 is as accurate as 2-band WBCK-3, and 4-band WBCK-3 displays better accuracy compared with 4-band WBCK-2, especially at low wall temperatures below about $500 \mathrm{~K}$.

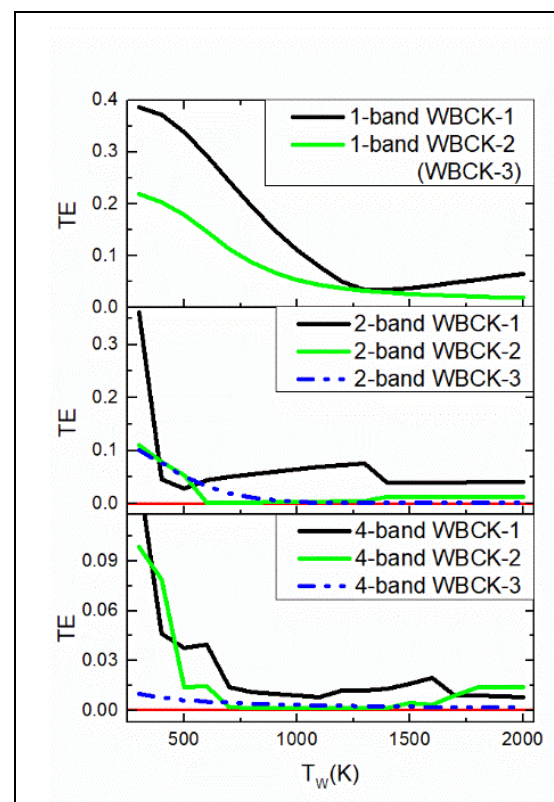

(a) BC-1

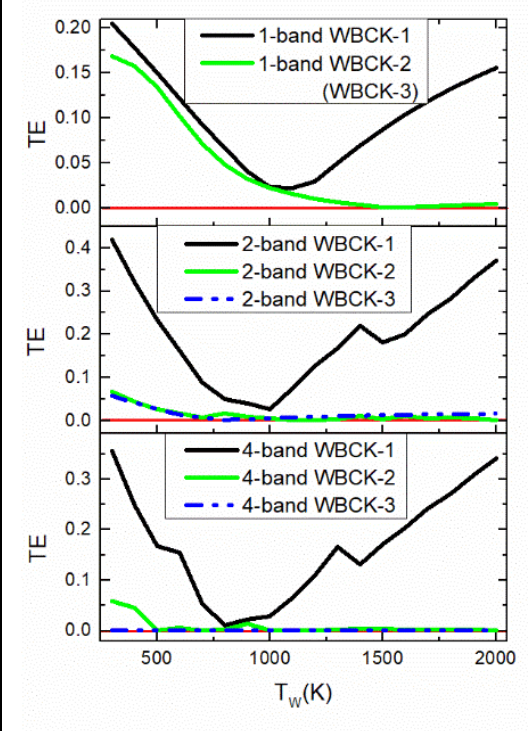

(b) BC-2

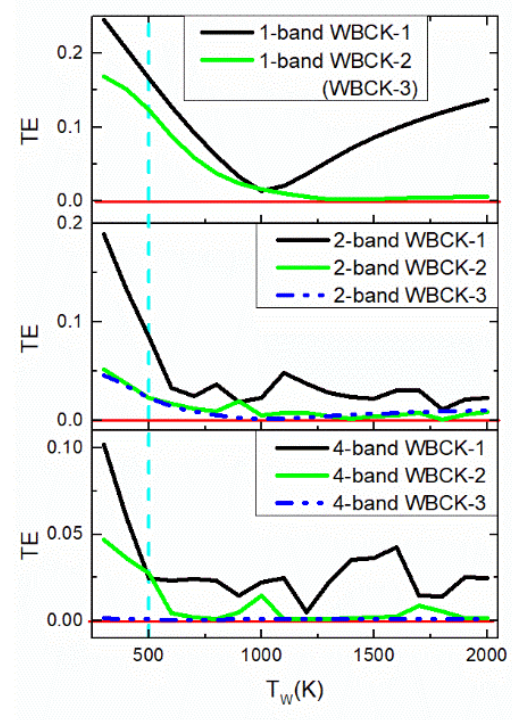

(c) BC-3

Fig. 4 Variation of TE predicted by different WBCK models and for different wall surface materials with the wall temperature for Case 1. 
Figure 5 shows the variation of EF of different models and surface materials with the wall temperature. For all the three surface materials, EF of 1-band WBCK-1 first decreases in magnitude with increasing temperature till reaching zero but then increases in magnitude with further increasing the wall temperature. On the other hand, EF of 1-band WBCK-2 (or WBCK3) decreases in magnitude with increasing temperature and then remains very low close to zero at wall temperatures above $1000 \mathrm{~K}$, regardless of the surface materials, as shown in the top row of Figure 5. Similar to the results shown in Figure 4, the $\mathrm{EF}$ values of 2- and 4-band WBCK-1 display different trends for different surface materials. For BC-1 and BC-3, the EF values of 2- and 4-band WBCK-1 first decrease in magnitude with increasing wall temperature and then fluctuate around zero at wall temperatures above about $800 \mathrm{~K}$. As shown in the middle and bottom rows of Figure 5(b), the EF values of 2- and 4band WBCK-1 for BC-2 increase nearly monotonically with increasing the wall temperature, similar to 1-band WBCK-1. The performance of 2- and 4-band WBCK-2 and WBCK-3 models evaluated in terms of EF is similar to that observed in TE shown in Figure 4. In summary, WBCK-1 is consistently less accurate than WBCK-2 and WBCK-3, regardless of the number of bands, wall temperature, or the type of wall materials, i.e., it is necessary to consider the spectral behavior of wall emissivity of the three typical wall materials. When the spectrum is divided into 2-bands, the accuracy of WBCK-2 and WBCK-3 is very similar. However, WBCK-3 is very accurate when the spectrum is divided into 4 wide-bands, regardless of the wall temperature or the wall material.

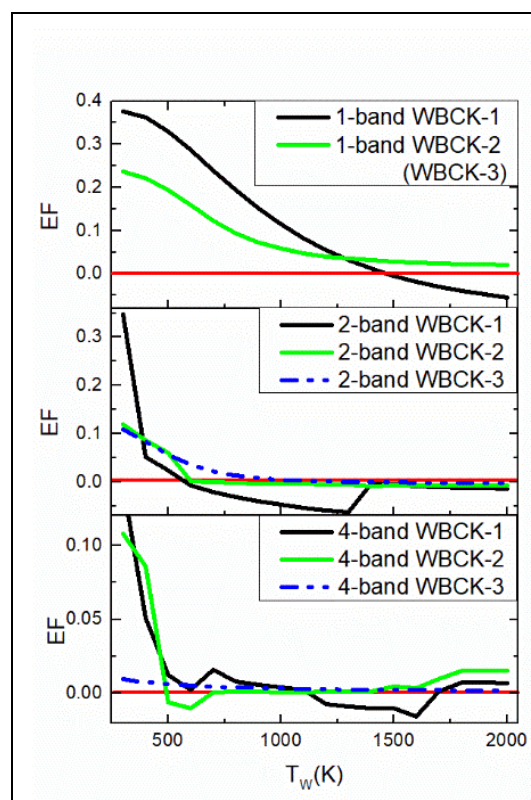

(a) BC-1

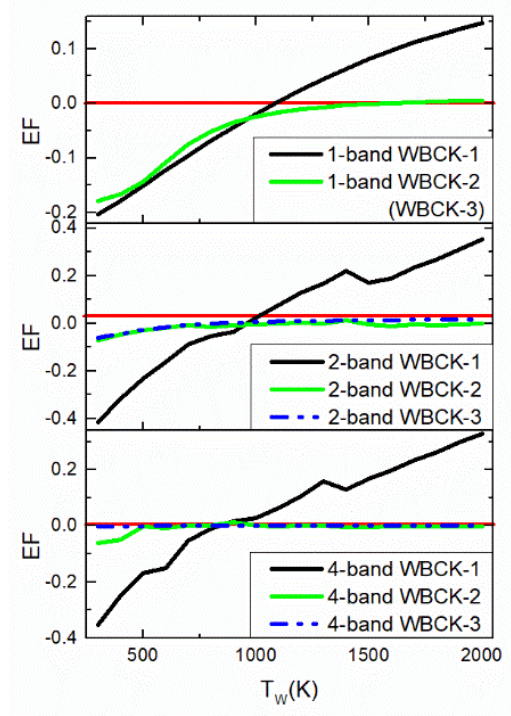

(b) BC-2

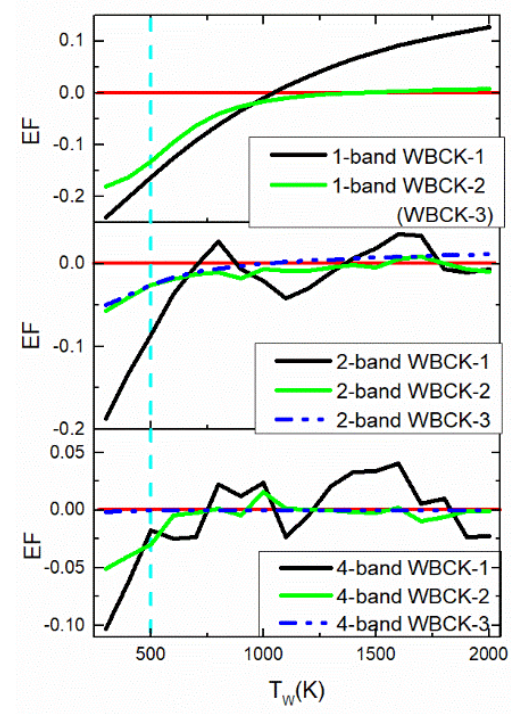

(c) BC-3

Fig. 5 Variation of EF predicted by different WBCK models and for different wall surface materials with the wall temperature for Case 1.

Figure 6 shows the distributions of radiative source term for wall temperature of $500 \mathrm{~K}$ and boundary condition of BC-3, corresponding to conditions marked by the vertical cyan dashed lines in Figures 4(c) and 5(c). Apart from the conclusions mentioned above, it can be seen that the 4-band WBCK-1 and WBCK-2 models show different trends of LE(x). To be more specific, the LE(x) values of WBCK-1 and WBCK-2 at the mid-point of computational domain are almost the same, while LE(x) of WBCK-1 and WBCK-2 near the wall are obviously different. This is the reason why the TE values of 4-band WBCK1 and WBCK-2 are almost identical, while the EF value of 4-band WBCK-1 is slightly smaller in magnitude than that of 4band WBCK-2. 


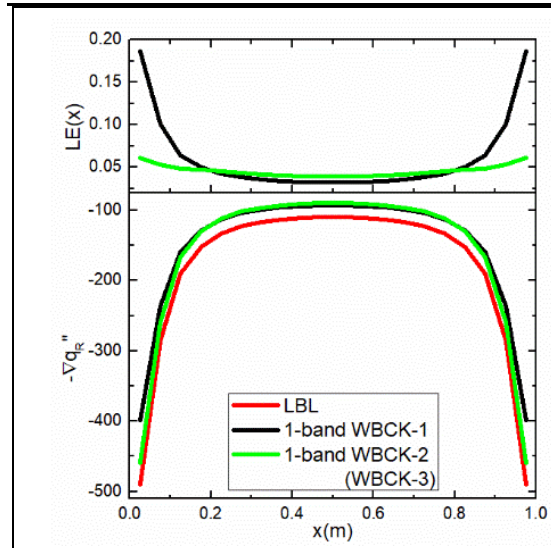

(a) 1-band WBCK

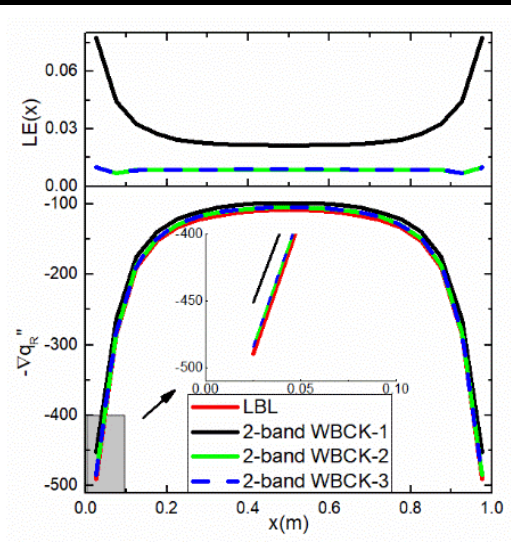

(b) 2-bands WBCK

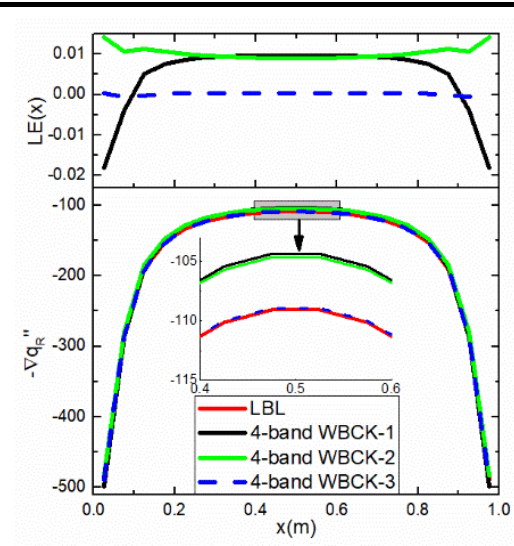

(c) 4-bands WBCK

Fig. 6 Distributions of the radiative source term of Case 1 for $500 \mathrm{~K}$ wall temperature with $\mathrm{BC}-3$ as the boundary material.

To explain why WBCK-2 is more accurate than WBCK-1, the left frame of Fig. 7 displays the 2nd-band spectral absorption coefficient of 2-band WBCK-1, the normalized spectral Planck function, $I_{b \eta} / I_{b}\left(\mathrm{~T}_{\mathrm{w}}=500 \mathrm{~K}\right)$, and the spectral emissivity of BC-3 as a function of wavenumber. The right frame of Fig. 7 shows the reordered absorption coefficient, $k^{*}$, and the 2 ndband reordered emissivity of WBCK-1 and WBCK-2 as a function of $g_{0}$. As is known, larger absorption coefficients are in general more important than smaller ones in radiative heat transfer, as shown in WBCK RTE (Eq. (4)). The spectral absorption coefficients of $\mathrm{CO}_{2}$ at the $4.3 \mu \mathrm{m}$ band (i.e., centered at $2410 \mathrm{~cm}^{-1}$ ) are much larger than those at other spectral positions [14], which means that the spectral emissivity near $2410 \mathrm{~cm}^{-1}$ has a great impact on the predicted radiative heat transfer. The gray horizontal dashed line marks the absorption coefficients larger than $0.05 \mathrm{~cm}^{-1}\left(\kappa\right.$ for the wavenumber-space and $k^{*}$ for the $g_{0}$-space). The cyan horizontal dashed lines mark the corresponding range of spectral emissivity, which is from 0.62 to 0.65 (see the left frame of Figure 7). The emissivity of WBCK-2 for absorption coefficients larger than $0.05 \mathrm{~cm}^{-1}$ is between 0.62 and 0.64 , and very close to the spectral emissivity in the wavenumber-space. As for WBCK-1, the emissivity of WBCK1 is 0.59 due to the influence of spectral Planck function weighting (see the red line of left frame of Figure 7). In other words, the emissivity of WBCK-2 is more accurate than that of WBCK-1, resulting in better accuracy of WBCK-2 than WBCK-1.

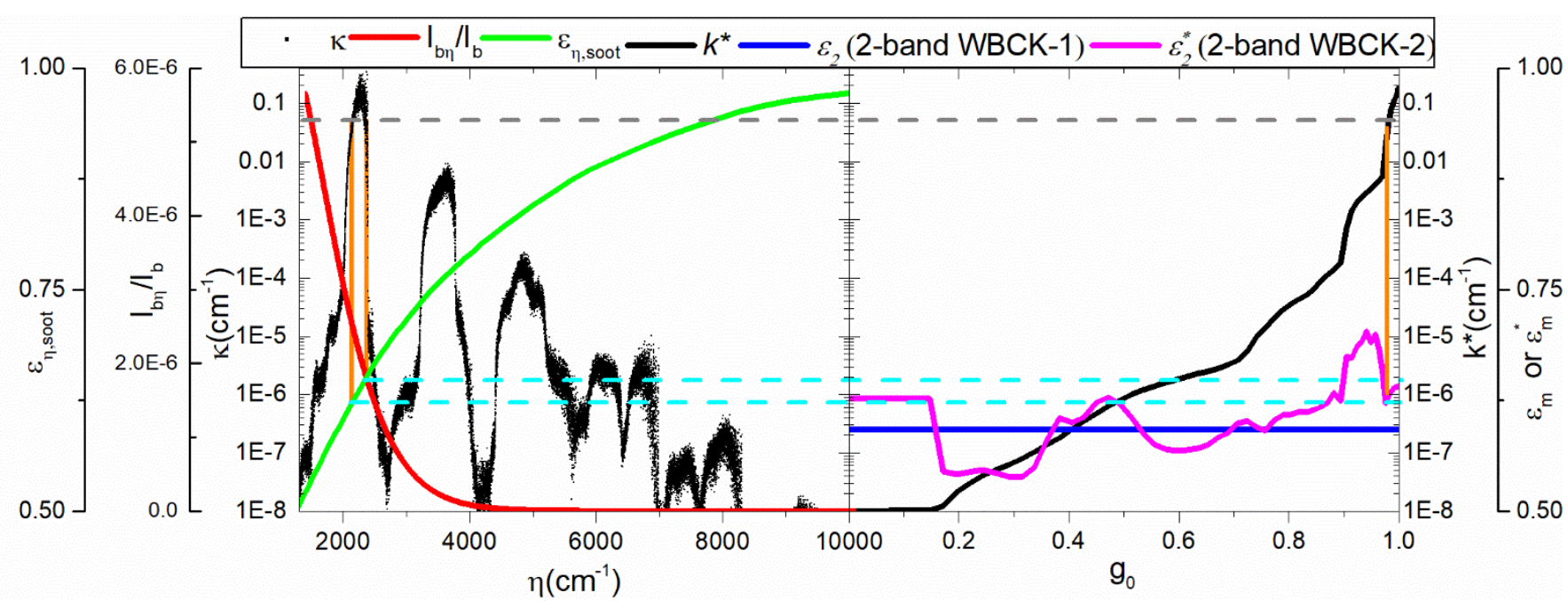

Fig. 7 The prediction of 2nd-band emissivity of two-band WBCK-1 and WBCK-2. The left frame displays the 2nd-band spectral absorption coefficient, the spectral Planck function and the spectral emissivity of BC-3. The right frame shows the reordered absorption coefficient and the 2nd-band emissivity of WBCK-1 and WBCK-2. 
remains nearly constant around $1 \%$. This is attributed to the different band intervals of WBCK-2 and WBCK-3. For WBCK2, the four bands are 200-900 $\mathrm{cm}^{-1}, 900-1400 \mathrm{~cm}^{-1}, 1400-2000 \mathrm{~cm}^{-1}$ and 2000-10000 $\mathrm{cm}^{-1}$, while for WBCK-3, the four bands are 200-1300 $\mathrm{cm}^{-1}, 1300-2700 \mathrm{~cm}^{-1}, 2700-4300 \mathrm{~cm}^{-1}$ and $4300-10000 \mathrm{~cm}^{-1}$.

Figure 8 displays the 4th-band emissivity of 4-band WBCK-2. The gray horizontal dashed lines mark absorption coefficients that lie between $0.001 \mathrm{~cm}^{-1}$ and $0.01 \mathrm{~cm}^{-1}$, and the cyan horizontal dashed line indicate the spectral emissivity of BC-3 (between 0.75 and 0.78 , see the left frame of Figure 8). However, the emissivity predicted by WBCK-2 is around 0.69, and is much smaller than the spectral emissivity in the wavenumber-space. Figure 9 shows the 3rd-band emissivity of 4-band WBCK-3. The emissivity of WBCK-3 ranges from 0.75 to 0.77 . In other words, the reordered emissivity predicted by WBCK3 shows better agreement with the spectral emissivity in the wavenumber-space than it predicted by WBCK-2. This explains why WBCK-3 is more accurate than WBCK-2 at a low wall temperature.

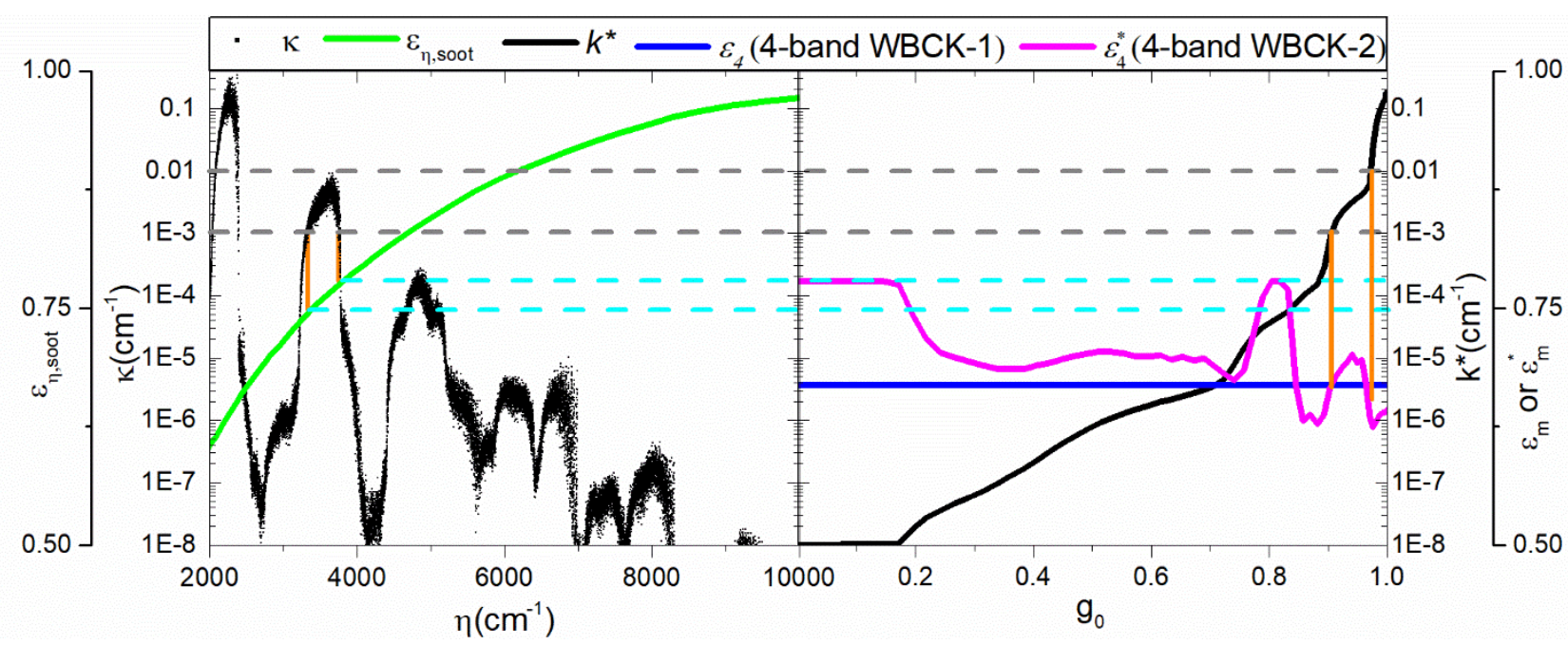

Fig. 8 The prediction of 4th-band emissivity of 4-band WBCK-1 and WBCK-2. The left frame displays the 4th-band spectral absorption coefficient and the spectral emissivity of BC-3. The right frame shows the reordered absorption coefficient and the 4th-band emissivity of WBCK-1 and WBCK-2.

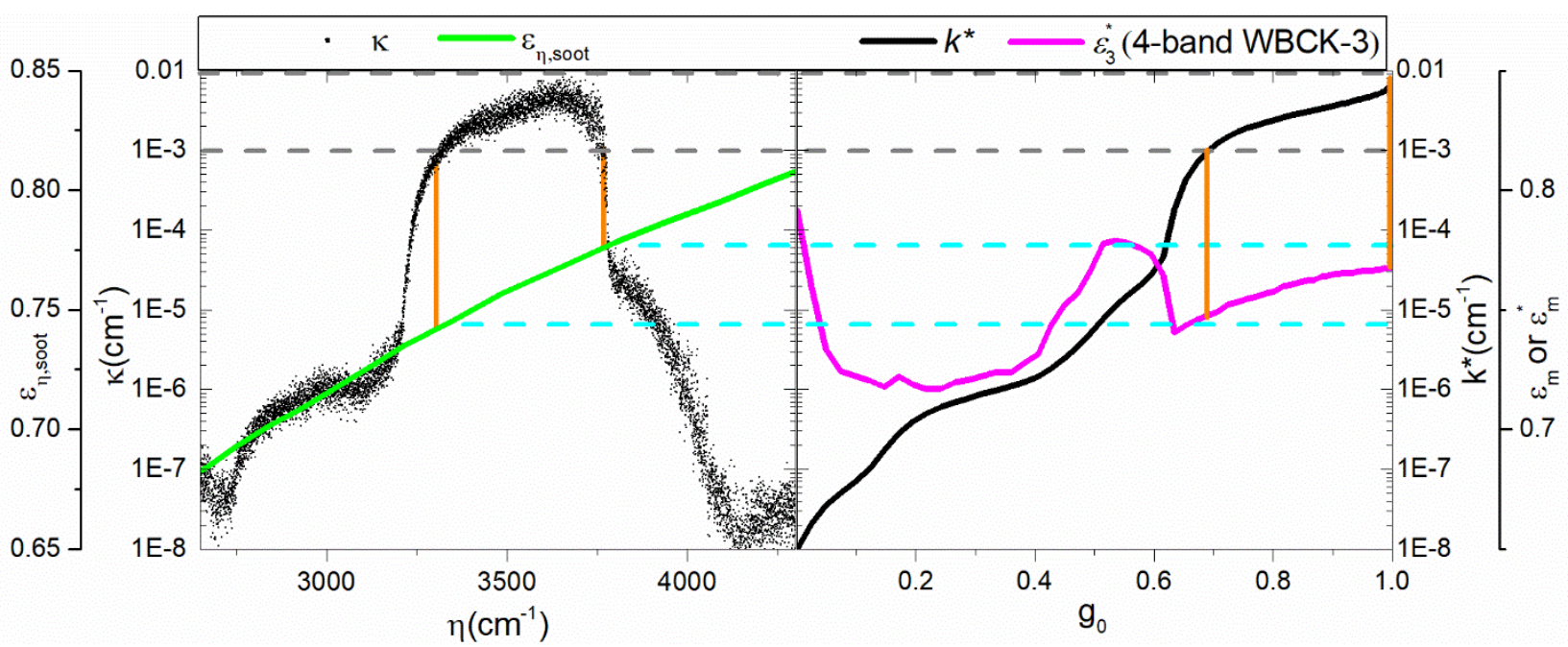

Fig. 9 The prediction of 3rd-band emissivity of 4-band WBCK-3. The left frame displays the 3rd-band spectral absorption coefficient and the spectral emissivity of BC-3. The right frame illustrates the reordered absorption coefficient and the 3rdband emissivity of WBCK-3.

\subsection{The pure $\mathrm{H}_{2} \mathrm{O}$ case}

The second test case (Case 2) contains pure $\mathrm{H}_{2} \mathrm{O}$ as the radiating gas, whose thermal conditions are given Table 1. The 
medium is composed of $10 \% \mathrm{H}_{2} \mathrm{O}$ (balanced by $\mathrm{N}_{2}$ ) at $2500 \mathrm{~K}$ at $1 \mathrm{~atm}$, while the wall temperature is varied from $300 \mathrm{~K}$ to $2000 \mathrm{~K}$. Figures 10 and 11 display the variations of TE and EF with the wall temperature. It can be seen that the trends of TE and EF of 1-band WBCK-1 and WBCK-2 (or WBCK-3) are consistent with those observed in Case 1, i.e., TE and EF of 1band WBCK-1 decrease in magnitude firstly as the wall temperature increases. After reaching the minimum, they increase in magnitude with further increasing the wall temperature. In contrast, TE and EF of 1-band WBCK-2 (or WBCK-3) decrease continuously with the increase of the wall temperature. Different from the pure $\mathrm{CO}_{2}$ case, 1-band WBCK-1 shows better accuracy than 1-band WBCK-2 (or WBCK-3) except for BC-2 and BC-3 with a high wall temperature. This may be attributed to the different radiative characteristics of $\mathrm{CO}_{2}$ and $\mathrm{H}_{2} \mathrm{O}$. Compared with $\mathrm{CO}_{2}$, which radiates predominately in four spectral regions [14], $\mathrm{H}_{2} \mathrm{O}$ radiates almost continuously over the entire spectrum.

The 2- and 4-band WBCK-1 models display different trends of TE and EF for different boundary materials, as in the pure $\mathrm{CO}_{2}$ case. For BC-1, both TE and EF of 2- and 4-band WBCK-1 first decrease sharply and then remain close to zero as the wall temperature further increases. For BC-2, as shown in the middle and bottom rows of Figures 10(b) and 11(b), TE and EF of 2- and 4-band WBCK-1 first decrease in magnitude then increase in magnitude with the wall temperature increases. As for BC-3, both TE and EF of 2- and 4-band WBCK-1 first decrease and then oscillate with increasing the wall temperature. In contrast, TE and EF of 2- and 4-band WBCK-2 and WBCK-3 decrease nearly monotonically in magnitude as the wall temperature increases regardless of the wall material, which are similar to Case 1.

Overall, the 2- and 4-band WBCK-2 models are more accurate than the corresponding WBCK-1 models, except when BC3 is the boundary material. For BC-3, the values of both TE and EF of 2- and 4-band WBCK-2 are almost the same as those of WBCK-1 when the wall temperature is lower than $1000 \mathrm{~K}$ and $700 \mathrm{~K}$, respectively. The 2-band WBCK-3 model displays lower accuracy than both WBCK-1 and WBCK-2; however, when the entire spectrum is divided into 4 bands, WBCK-3 is more accurate than WBCK-1 and WBCK-2.

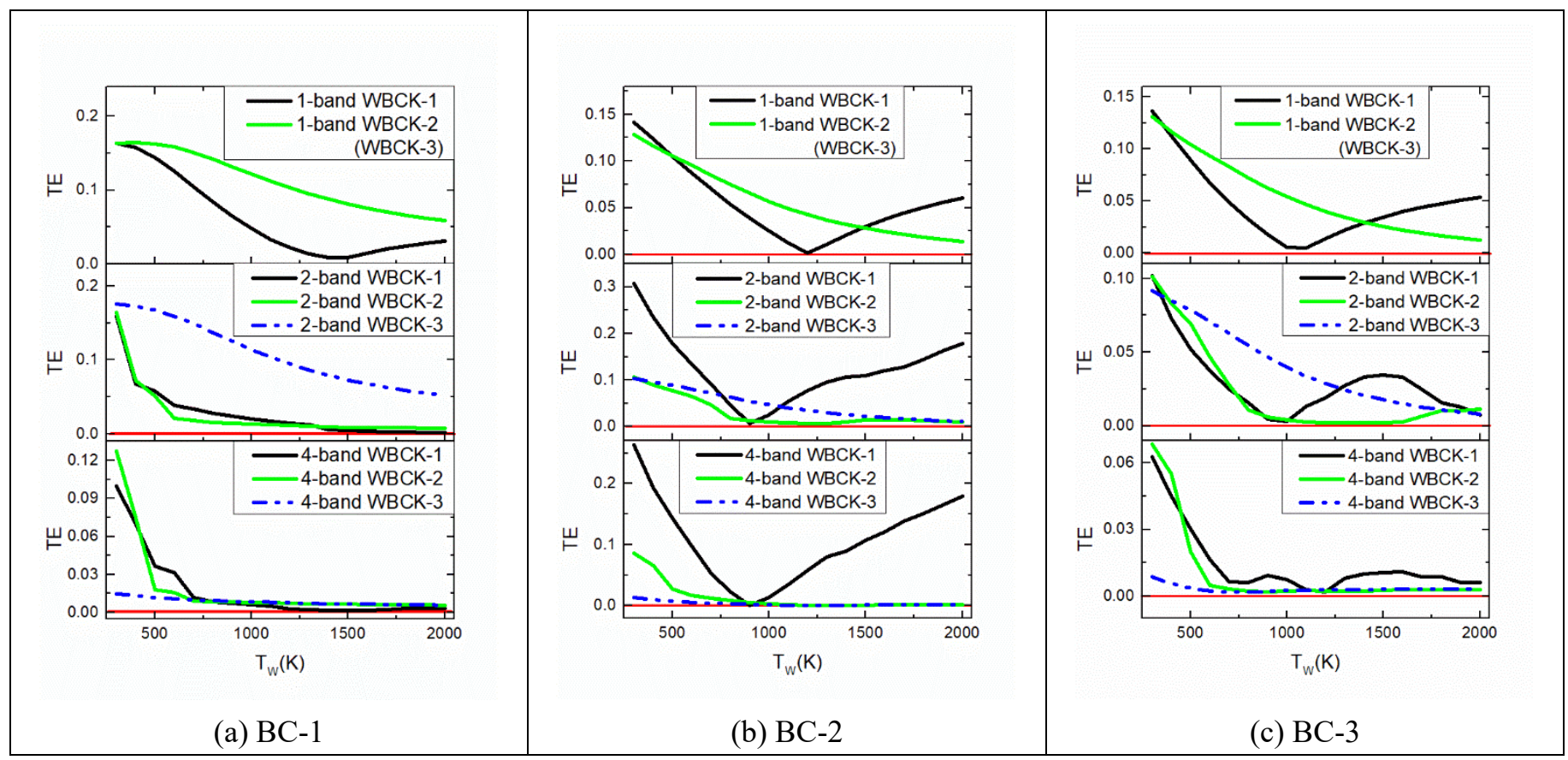

Fig. 10 Variation of TE predicted by different WBCK models and for different wall surface materials with the wall temperature for Case 2. 


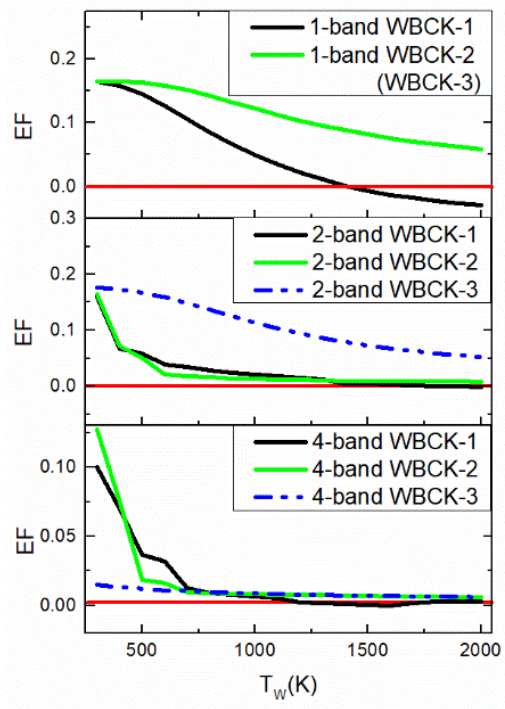

(a) BC-1

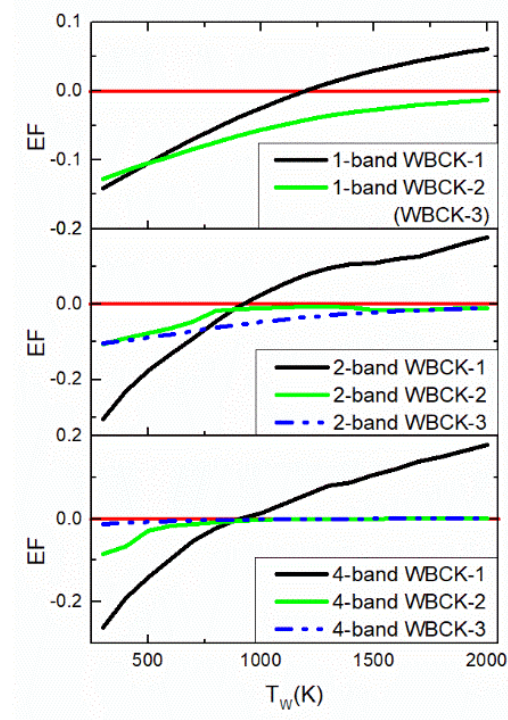

(b) BC-2

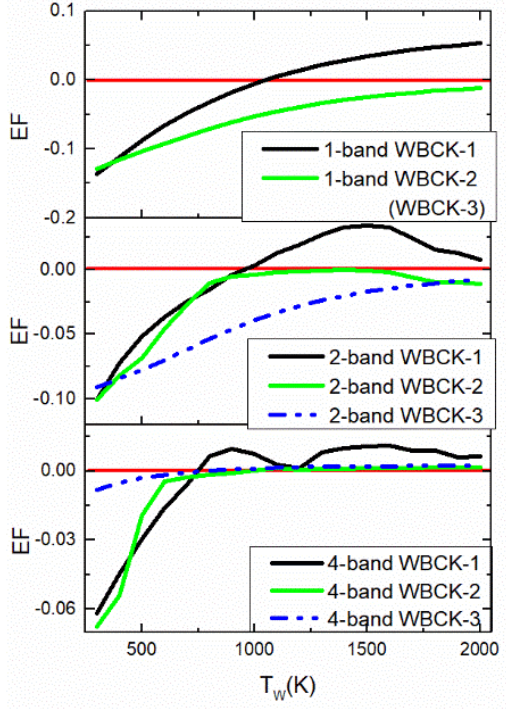

(c) $\mathrm{BC}-3$

Fig. 11 Variation of EF predicted by different WBCK models and for different wall surface materials with the wall temperature for Case 2 .

\subsection{The $\mathrm{CO}_{2}-\mathrm{H}_{2} \mathrm{O}$ mixture case}

In the final 1D test case (Case 3), the medium is a $\mathrm{CO}_{2}-\mathrm{H}_{2} \mathrm{O}$ mixture and the thermal conditions are also given in Table 1. The gaseous medium consists of $10 \% \mathrm{CO}_{2}$ and $20 \% \mathrm{H}_{2} \mathrm{O}$ (balanced by $\mathrm{N}_{2}$ ) at $2500 \mathrm{~K}$ and $1 \mathrm{~atm}$, while the wall temperature is varied from $300 \mathrm{~K}$ to $2000 \mathrm{~K}$. Figures 12 and 13 display the variations of TE and EF with the wall temperature. The trends of TE and EF of 1-, 2- and 4-band WBCK-1, WBCK-2 and WBCK-3 are consistent with those observed in Case 2. The performances of 1-, 2- and 4-band WBCK-1, WBCK-2 and WBCK-3 are also similar to those in the pure $\mathrm{H}_{2} \mathrm{O}$ case (Case 2) as well. In other words, 1-band WBCK-1 is more accurate than 1-band WBCK-2 (or WBCK-3), the 2- and 4-band WBCK-2 models demonstrate better accuracy than the 2- and 4-band WBCK-1 models, and the 2-band WBCK-3 model is less accurate than the 2-band WBCK-2 model; however, WBCK-3 can become very accurate when the entire spectrum is divided into four bands, especially at low wall temperatures.

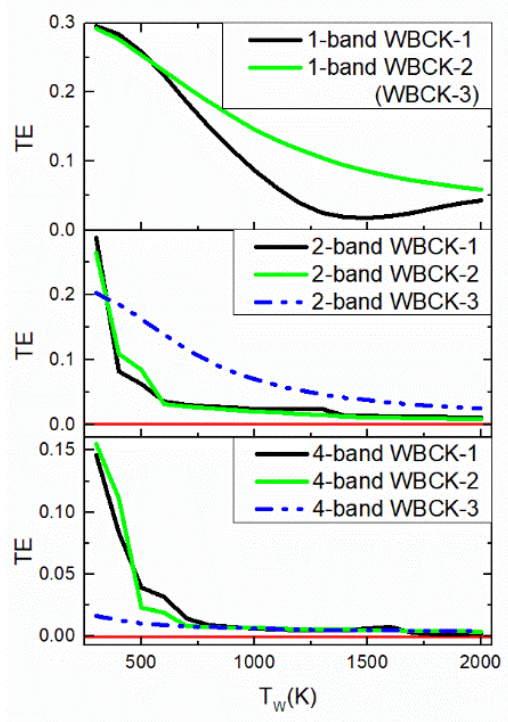

(a) BC-1

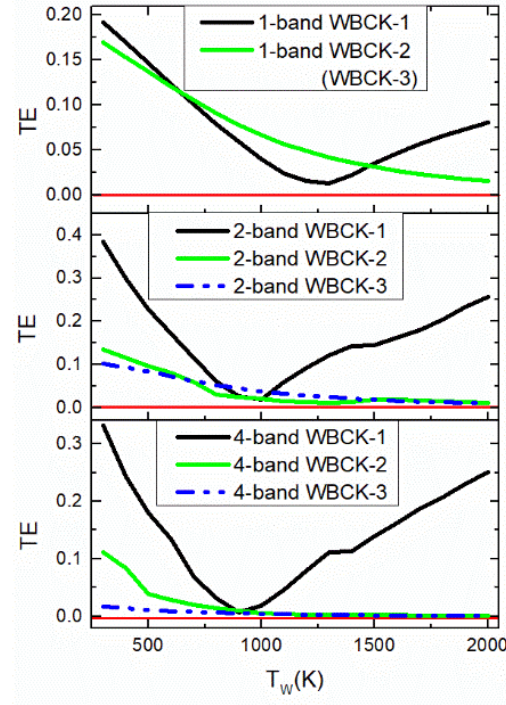

(b) BC-2

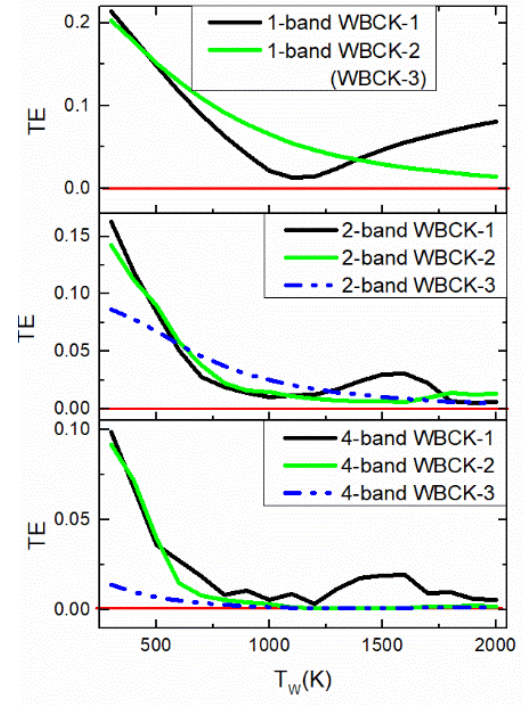

(c) BC-3

Fig. 12 Variation of TE predicted by different WBCK models and for different wall surface materials with the wall 
temperature for Case 3 .

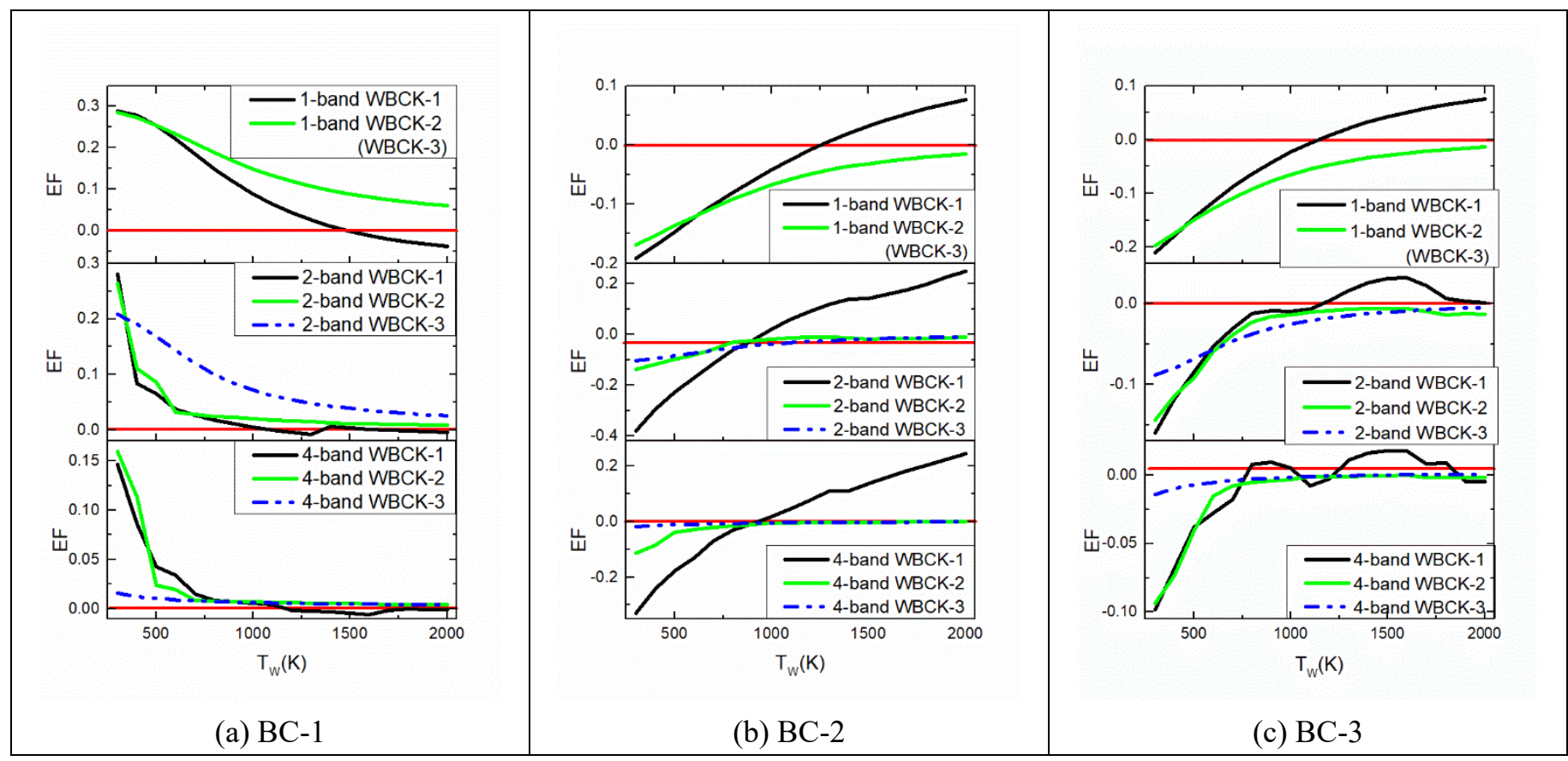

Fig. 13 Variation of EF predicted by different WBCK models and for different wall surface materials with the wall temperature for Case 3.

\subsection{The influence of number of bands}

As displayed above, WBCK-3 shows worse accuracy than WBCK-2 when the spectrum is divided into 2 bands; however, it displays significantly better accuracy when the number of bands reaches 4 . In this section, the influence of the number of bands is investigated for Case 3 at the wall temperature of $300 \mathrm{~K}$ and $1500 \mathrm{~K}$.

Figure 14 and 15 shows the variation of TE with the number of bands. It can be seen that, for BC-1 and BC-3 at wall temperature of $300 \mathrm{~K}$, TE of both WBCK-1 and WBCK-2 decreases with increasing the number of bands, though somewhat gradually, while it displays much weaker dependence on the number of bands for BC-2. TE of WBCK-3 drops rapidly with increasing the number of bands from 1 to 3 for all the three wall materials and then remains close to zero as the number of bands further increases beyond 3 .

For the wall temperature of $1500 \mathrm{~K}$, the TE trends of WBCK-1 are the same as it performed in Fig. 14. In contrast, the TE trends of WBCK-2 and WBCK-3 are almost consistent with those of WBCK-3 in Fig. 14, i.e., TE of WBCK-2 and WBCK-3 decreases sharply as the number of bands increases, and they are negligible when the number of bands exceeds 3 or 4 .

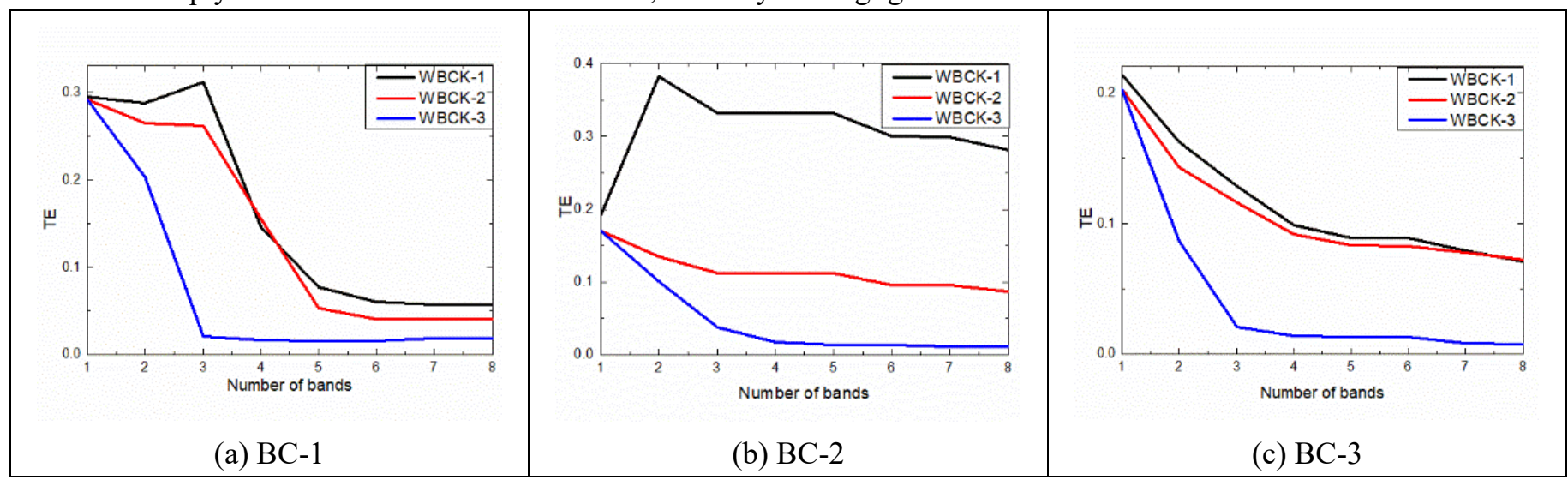

Fig. 14 Variation of TE with the number of bands for Case 3, in which the wall temperature is $300 \mathrm{~K}$. 


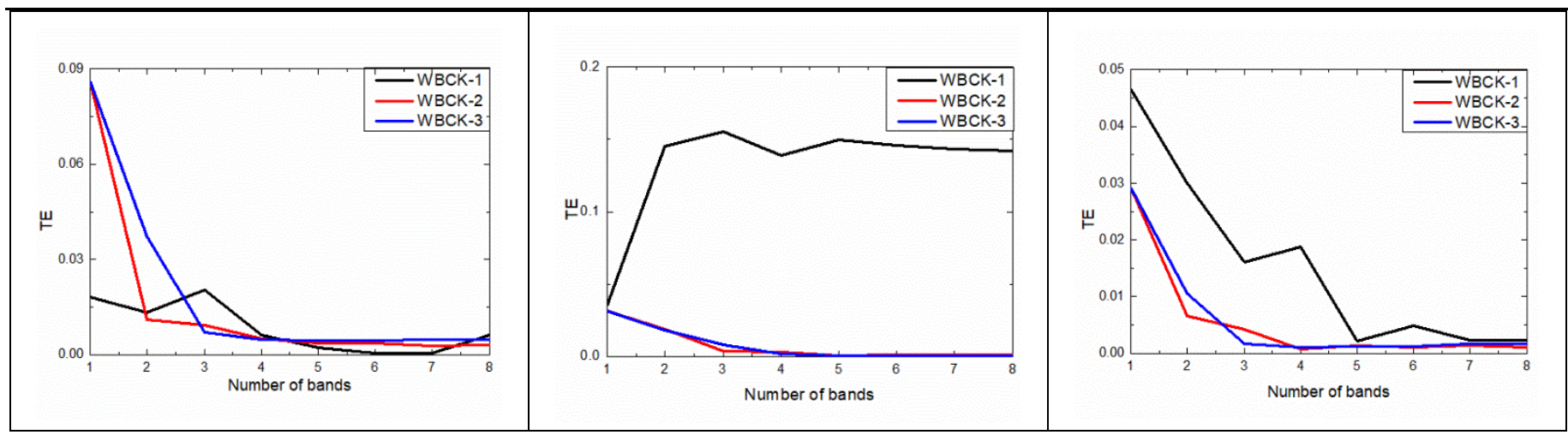

Fig. 15 Variation of TE with the number of bands for Case 3, in which the wall temperature is $1500 \mathrm{~K}$.

\section{Results and discussion of a three-dimensional fuel-air flame}

In this section, the accuracy of 1- and 4-band WBCK-1, WBCK-2 and WBCK-3 in a 3D enclosure containing a fuel-air flame [19], [], ] is evaluated. The combustion chamber is a rectangular enclosure of $2 \mathrm{~m} \times 2 \mathrm{~m} \times 4 \mathrm{~m}$. The wall temperature is set to $300 \mathrm{~K}$ [31], and BC-1 is used as the wall material here, which is different from Refs. [19], [], ]. A mixture of $10 \%$ $\mathrm{CO}_{2}, 20 \% \mathrm{H}_{2} \mathrm{O}$ and $70 \% \mathrm{~N}_{2}$ is uniformly distributed in the combustion chamber. The temperature profile within the combustion chamber is axisymmetric, which is given by Equations (22) and (23)

$T=\left(T_{c}-T_{e}\right) f\left(r_{c} / R\right)+T_{e}$

$f\left(r_{c} / R\right)=1-3\left(r_{c} / R\right)^{2}+2\left(r_{c} / R\right)^{3}$

where $T_{c}$ is the temperature along the centerline $(0,0, \mathrm{z}), T_{e}$ is the temperature at the exit of combustion chamber $(z=4 \mathrm{~m})$, $R$ is the radius of $1 \mathrm{~m}$. The temperature along the centerline of combustion chamber increases linearly from $400 \mathrm{~K}$ at $z=0$ to $1800 \mathrm{~K}$ at $z=0.375 \mathrm{~m}$, then it decreases linearly to the $800 \mathrm{~K}$ at $z=4 \mathrm{~m}$. The gas temperature in the corner regions, where $r_{\mathrm{c}}>R$, is set to the exit temperature of $T_{\mathrm{e}}=800 \mathrm{~K}$.

The number of computational grids is $17 \times 17 \times 24$. The grid is uniformly distributed in the $\mathrm{x}$ and $\mathrm{y}$ directions, and nonuniformly distributed in the $\mathrm{z}$ (streamline) direction. The details of grid are consistent with those used in Liu [31]. DOM with the $\mathrm{T}_{4}$ [33] angular discretization scheme is used to solve RTE. NBCK with band interval of $50 \mathrm{~cm}^{-1}$ is used as the benchmark because the computational resources required by LBL in this 3D case are unacceptably large The 8-point and 16-point GaussChebyshev quadrature schemes are used for the NBCK and WBCK methods, respectively. The emission-weighted temperature is chosen as the reference temperature when using the WBCK method [34].

Figure 16 displays the radiative source terms along the enclosure centerline $(0,0, \mathrm{z})$ predicted by NBCK (bounded by black walls and BC-1), 1-band WBCK-1, 1-band WBCK-2, and 4-band WBCK-1, WBCK-2 and WBCK-3. It can be found that the wall material (black or non-black) has some effects on the radiative source term near the wall, as shown in Figure 16(a) (compare the results of NBCK with the black walls and BC-1). The results of 1-band WBCK-1 and WBCK-2 are almost the same, as already observed in Figure 12, and the maximum LE(x) in magnitude is about $-8.7 \%$ and $-8.8 \%$. The predictions of 4-band WBCK-1 and WBCK-2 are also almost the same, Fig. 16(b), which is also similar to the results shown in Fig. 12. When using 4 bands, WBCK-3 can effectively improve the accuracy of WBCK-1 and WBCK-2. The maximum LE(x) in magnitude of WBCK-1, WBCK-2 and WBCK-3 are $-8.8 \%,-9.0 \%$ and $-1.2 \%$, respectively, as shown in the top frame of Fig. 16(b). 


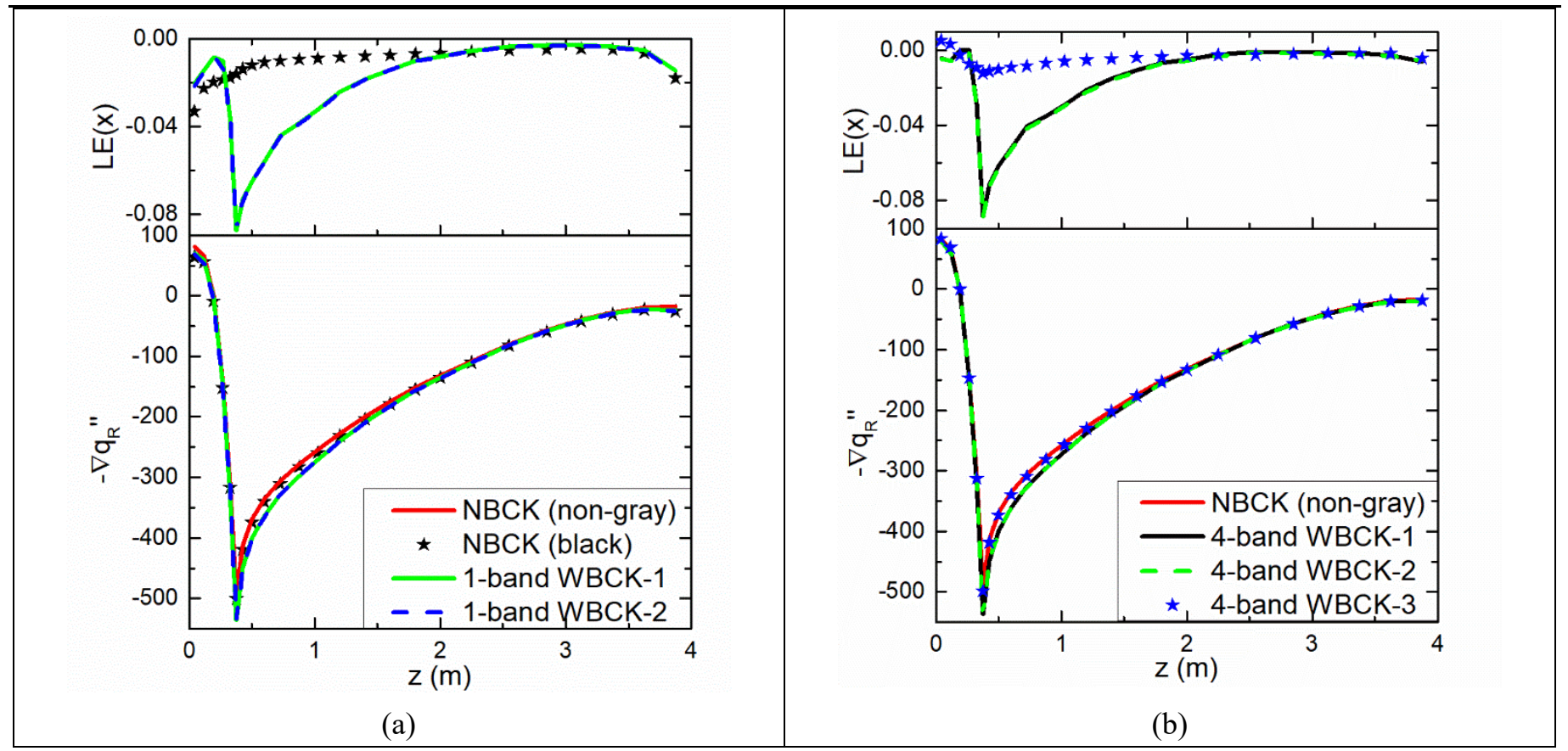

Fig. 16 The radiative source term distributions along the enclosure centerline $(0,0, \mathrm{z})$ of the fuel-air flame predicted by NBCK (bounded by the black walls and BC-1), 1-band WBCK-1, 1-band WBCK-2, and 4-band WBCK-1, WBCK-2 and WBCK-3.

Figure 17 displays the net heat fluxes along the centerline of a side wall, $(1 \mathrm{~m}, 1 \mathrm{~m}, \mathrm{z})$, by NBCK (bounded by the black walls and BC-1), 1-band WBCK-1, 1-band WBCK-2, and 4-band WBCK-1, WBCK-2 and WBCK-3. There are obvious discrepancies between the NBCK results for the two boundary conditions (black wall vs. BC-1). For the black walls, the maximum flux along the line of $(1 \mathrm{~m}, 1 \mathrm{~m}, \mathrm{z})$ is $20.5 \mathrm{kw} / \mathrm{m}^{2}$, which is consistent with the result of Porter et al. [32], while for $\mathrm{BC}-1$, the maximum net flux is lower at $16.1 \mathrm{kw} / \mathrm{m}^{2}$. Similar to the radiative source term along the center line $(0,0, \mathrm{z}), 1$-band WBCK-1 and WBCK-2 predicted undistinguishable results but with large errors. When using 4 bands, WBCK-1 and WBCK2 yield significantly better accuracy than their 1-band counterparts and their results are very close to each other as well. When the spectrum is divided into 4 bands, WBCK-3 offers the best accuracy among the three WBCK models investigated.

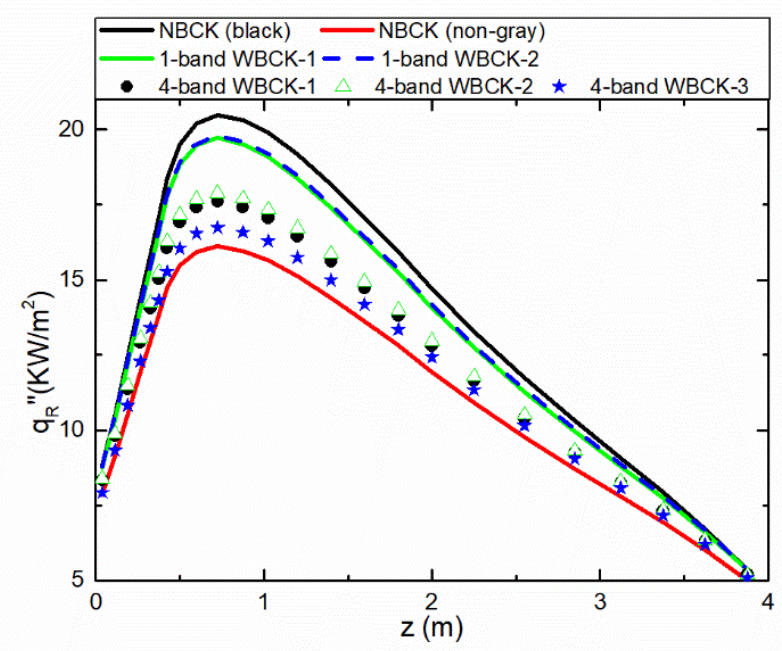

Fig. 17 The radiative heat flux along the line of $(1 \mathrm{~m}, 1 \mathrm{~m}, \mathrm{z})$ predicted by NBCK (bounded by the black walls and BC-1), 1-band WBCK-1, 1-band WBCK-2, and 4-band WBCK-1, WBCK-2 and WBCK-3.

\section{Conclusions}

In this work, a nongray-wall emissivity model is proposed within the framework of the Wide-Band Correlated K- 
distribution method. In addition, an absorption-coefficient-based strategy is developed to optimize the band intervals. Three 1D isothermal and homogeneous cases bounded by the fly-ash deposit, GH536, and soot deposit and a 3D fuel-air flame bounded by a rectangular enclosure bounded by fly-ash deposit coated walls are considered as test cases to evaluate the accuracy of WBCK-1, WBCK-2 and WBCK-3 for different numbers of bands up to 4 in the prediction of radiative heat transfer. The LBL and NBCK results are obtained and used as the benchmarks in the one- and three-dimensional cases, respectively. The following conclusions can be drawn from this work:

(1) WBCK-2 improves the accuracy of WBCK-1 when the number of bands is larger than 1. For 1-band WBCK (i.e., FSCK), WBCK-2 is more accurate than WBCK-1 in the pure $\mathrm{CO}_{2}$ case, while they show the opposite trends in the pure $\mathrm{H}_{2} \mathrm{O}$ and $\mathrm{CO}_{2}-\mathrm{H}_{2} \mathrm{O}$ case.

(2) When the entire spectrum is divided into 2 bands, WBCK-3 demonstrates the worst accuracy among the models and thermal conditions investigated.

(3) When the spectrum is divided into 4 bands, WBCK-3 is more accurate than WBCK-1 and WBCK-2 for low-temperature wall. For high-temperature wall, both WBCK-2 and WBCK-3 agree well with the benchmark.

(4) For the three types of typical wall material of nongray emissivity encountered in practical combustion systems, it is sufficient to divide the entire spectrum into 3 or 4 bands when using WBCK-3 for accurate predictions of radiative heat transfer.

\section{Acknowledgments}

The authors are grateful for the former support of "National Natural Science Foundation of China "(Grant No.50606004) on the radiative heat transfer modelling topic, and the support of "Overseas Expertise Introduction Project for Discipline Innovation ”(111 project, Grant No. B08009) for international academic exchanges.

\section{Reference}

[1] Modest M F, Haworth D C. Radiative heat transfer in turbulent combustion systems: theory and application. New York: Springer; 2016.

[2] Wall T F, Bhattacharya S P, Baxter L L, Richards G, Harb J N. The character of ash deposits and the thermal performance of furnaces. Fuel Process Technol 1995; 44(1): 143-153.

[3] Dannecker R, Schildmacher K U, Noll B, Koch R, Hase M, Krebs W, Aigner M. Impact of radiation on the wall heat load at a test bench gas turbine combustion chamber: measurements and CFD simulation. ASME Turbo Expo 2007; 4: 1311-1321.

[4] Ren T, Modest M F, Roy S. Monte carlo simulation for radiative transfer in a high-pressure industrial gas turbine combustion chamber. J Eng Gas Turb Power 2018; 140(5): 051503.

[5] Goody R M. A statistical model for water-vapor absorption, Quart J R Meteorol Soc 1952; 78: 165-169.

[6] Malkmus W. Random Lorentz band model with exponential-tailed $\mathrm{S}^{-1}$ line-intensity distribution function. J Opt Soc Am 1967; 57: 323-329.

[7] Lacis A A, Oinas V. A description of the correlated $\mathrm{k}$ distribution method for modeling non-gray gaseous absorption, thermal emission, and multiple scattering in vertically inhomogeneous atmospheres. J Geophys Res 1991; 96: 90279063.

[8] Modest M F, Narrow-band and full-spectrum k-distributions for radiative heat transfer-correlated-k vs. scaling approximation. J Quant Spectrosc Radiat Transf 2003; 76: 69-83.

[9] Ströhle J, Coelho P J. On the application of the exponential wide band model to the calculation of radiative heat transfer in one- and two-dimensional enclosures. Intl J Heat Mass Transf 2002; 45(10): 2129-2139.

[10] Ströhle J. Assessment of the re-ordered wide band model for non-grey radiative transfer calculations in 3D enclosures. J Quant Spectrosc Radiat Transf 2008; 109(9): 1622-1640.

[11] Denison M K, Webb B W. A spectral line-based weighted sum of gray gases model for arbitrary RTE solvers. ASME J Heat Transf 1993; 115(4): 1004-1012.

[12] Pierrot L, Rivière P, Soufiani A, Taine J. A fictitious-gas-based absorption distribution function global model for radiative 
transfer in hot gases. J Quant Spectrosc Radiat Transf 1999; 62(5): 609-624.

[13] Johansson R, Leckner B, Andersson K, Johnsson F. Account for variations in the $\mathrm{H}_{2} \mathrm{O}$ to $\mathrm{CO}_{2}$ molar ratio when modelling gaseous radiative heat transfer with the Weighted-Sum-of-Grey-Gases Model. Combust Flame 2011; 158(5): 893-901.

[14] Modest M F. Radiative heat transfer. 3rd ed.. New York: Academic Press; 2013.

[15] Chu H, Ren F, Feng Y, Gu M, Zheng S. A comprehensive evaluation of the non gray gas thermal radiation using the lineby-line model in one- and two-dimensional enclosures. Appl Therm Eng 2017; 124: 362-370.

[16] Solovjov V P, Lemonnier D, Webb B W. Efficient cumulative wavenumber model of radiative transfer in gaseous media bounded by non-gray walls. J Quant Spectrosc Radiat Transf 2013; 128: 2-9.

[17] Fonseca R J C, Fraga G C, Silva R B, Franca F H R. Application of the WSGG model to solve the radiative transfer in gaseous systems with nongray boundaries. ASME J Heat Transf 2018; 140(5): 052701.

[18] Bordbar H, Hyppänen T. Line by line based band identification for non-gray gas modeling with a banded approach. Int J Heat Mass Tran 2018; 127: 870-884.

[19] Bordbar H, Maximov A, Hyppänen T. Improved banded method for spectral thermal radiation in participating media with spectrally dependent wall emittance. Appl Energ 2019; 235: 1090-1105.

[20] Fonseca R J C, Fraga G C, Franca F H R. A simplified approach to compute the radiative transfer in a participating media bounded by non-gray walls. Eurotherm Seminar 110-Computational Thermal Radiation in Participating Media-VI; 2018.

[21] Liu Y, Liu G, Hu H, Kong B. A wavenumber subinterval grouping strategy compatible with non-gray metal wall boundaries used in multi-scale multi-group full spectrum k-distribution gas radiation model. Appl Therm Eng 2019; 113: 20-26.

[22] Zhao X Y, Haworth D C, Ren T, Modest M F. A transported probability density function/photon Monte Carlo method for high-temperature oxy-natural gas combustion with spectral gas and wall radiation. Combust Theor Model 2013; 17(2): 354-381.

[23] Yang X, He Z, Dong S, Tan H. Prediction of turbulence radiation interactions of $\mathrm{CH}_{2}-\mathrm{H}_{2} /$ air turbulent flames as atmospheric and elevated pressures. Int J Hydrogen Energ 2018; 43(32): 15537-15550.

[24] Johansson R, Leckner B, Andersson K, Johnsson F. Influence of particle and gas radiation in oxy-fuel combustion. Int J Heat Mass Tran 2013; 65: 143-152.

[25] Kong B, Li T, Eri Q. Normal spectral emissivity of GH536 (HastelloyX) in three surface conditions. Appl Therm Eng 2017; 113: 20-26.

[26] Markham J L, Best P E, Solomon P R, Yu Z Z. Measurement of radiative properties of ash and slag by FT-IR emission and reflection spectroscopy. ASME J Heat Transf 1992; 114(2): 458-64.

[27] Liebert C H, Hibbard R R. Spectral emittance of soot. NASA Technical Notes 1970;

[28] Hu H, Wang Q. Improved spectral absorption coefficient grouping strategy of wide band k-distribution model used for calculation of infrared remote sensing signal oh hot exhaust systems. J Quant Spectrosc Radiat Transf 2018; 213: 17-34.

[29] Rothman L S, Gordon I E, Barber R J, Dothe H, Gamache R R, Goldman A, Perevalov V I, Tashkun S A, Gamache J. HITEMP, the high-temperature molecular spectroscopic database. J Quant Spectros Radiat Transf 2010; 111: 2139-2150.

[30] Kochanov R V, Gordon I E, Rothman L S, Wcislo P, Hill C, Wilzewski J S. HITRAN application programming interface (HAPI): a comprehensive approach to working with spectroscopic data. J Quant Spectrosc Radiat Transf 2016; 177: 15 30.

[31] Liu F. Numerical solutions of three-dimensional non-grey gas radiative transfer using the statistical narrow-band model. ASME J Heat Transfer 1999; 121(1): 200-203.

[32] Porter R, Liu F, Pourkashanian M, Williams A, Smith D. Evaluation of solution methods for radiative heat transfer in gaseous oxy-fuel combustion environments. J Quant Spectrosc Radiat Transfer 2010; 111(14): 2084-2094.

[33] Thurgood C P, Becker H A, Pollard A. The $\mathrm{T}_{\mathrm{N}}$ quadrature set for the discrete-ordinates method. ASME J Heat Transfer 1995; 117: 1068-1070.

[34] Modest M F, Zhang H. The full-spectrum correlated-k distribution for thermal radiation from molecular gas-particulate mixtures. ASME J Heat Transf 2002; 124(1): 30-38. 